\title{
Ruthenium-Catalyzed Urea Synthesis Using Methanol as the C1 Source
}

Seung Hyo Kim and Soon Hyeok Hong*

Center for Nanoparticle Research, Institute for Basic Science (IBS), Seoul 151-742, Republic of Korea

Department of Chemistry, College of Natural Sciences, Seoul National University, Seoul 151-747, Republic of Korea

Korea Carbon Capture Sequestration R\&D Center (KCRC), Daejeon 305-303, Republic of Korea

E-mail: soonhong@snu.ac.kr

\section{Table of Contents}

Contents:

General Information

General Procedure for Symmetrical Urea Synthesis

General Procedure for Unsymmetrical Urea Synthesis

Characterization Data

Complementary Reaction Optimization Data

LC Chart

References

NMR Spectra 


\section{General Information}

Unless otherwise noted, all reactions were carried out using standard Schlenk techniques or in an argon-filled glove box. All symmetrical urea synthesis were performed in an oven-dried stainless steel vessel under argon atmosphere (internal pressure of the reaction vessel was increased to about 3 bar during the reaction at $140{ }^{\circ} \mathrm{C}$ ). In case of unsymmetrical urea synthesis a thick-wall glass tube was used with a safety shield. Ru complexes $\mathbf{3}^{1}$ and $\mathbf{4}^{2}$ were prepared according to the literature procedures. Unless otherwise noted, all reagents were obtained from Alfa Aesar, Strem, and Sigma Aldrich used as received. Analytical TLC was performed on a Merck 60 F254 silica gel plate (0.25mm thickness). Column chromatography was performed on Merck 60 silica gel (230-400 mesh). NMR spectra were recorded on a Bruker DPX-300 (300 MHz) spectrometer. Tetramethysilane was used as reference, and the chemical shifts were reported in ppm and the coupling constant in Hz. Chiral compounds analysis were carried out on an Agilent LC chromatograph using CHIRALPAK AS-H column (particle size $5 \mu \mathrm{m}$, dimensions $4.6 \mathrm{~mm} \times 250 \mathrm{~mm}$ ). HRMS analysis was performed by Organic Chemistry Research Center in Sogang University.

\section{General Procedure for Symmetrical Urea Synthesis}

A stainless steel autoclave $(50 \mathrm{~mL})$ was charged with complex $1(0.01 \mathrm{mmol}, 5.9 \mathrm{mg})$, amine (2 mmol), methanol $(2 \mathrm{mmol}, 81 \mu \mathrm{L})$, and toluene $(1.5 \mathrm{~mL})$ in an argon-filled glove box. The vessel was heated to $140{ }^{\circ} \mathrm{C}$ (bath temperature) under closed conditions. After $24 \mathrm{~h}$, the reaction was cooled to $0{ }^{\circ} \mathrm{C}$ for $30 \mathrm{~min}$. The generated $\mathrm{H}_{2}$ was carefully released in a fumehood. $n$-Hexane $(40 \mathrm{~mL})$ was then added to the reaction mixture. The resulting solid was collected and further washed with cold diethyl ether to afford the corresponding product. 


\section{General Procedure for Unsymmetrical Urea synthesis}

A Schlenk-type thick-wall round-bottom flask $(50 \mathrm{~mL})$ was charged with complex $1(0.01 \mathrm{mmol}$, $5.9 \mathrm{mg})$, benzyl amine $(0.5 \mathrm{mmol}, 54 \mathrm{mg})$, and methanol $(2 \mathrm{~mL})$ in an argon-filled glove box. The reaction tube was heated to $150{ }^{\circ} \mathrm{C}$ (bath temperature) under closed conditions. After $12 \mathrm{~h}$, the reaction was cooled to $0{ }^{\circ} \mathrm{C}$ for $1 \mathrm{~h}$. Methanol was removed under vacuum. Complex $\mathbf{1}(0.017 \mathrm{mmol}$, $9.9 \mathrm{mg})$, amine $(0.42 \mathrm{mmol})$, and toluene $(1.5 \mathrm{~mL})$ were then added to the reaction tube under an argon atmosphere. The vessel was heated to $120{ }^{\circ} \mathrm{C}$ (bath temperature) for $16 \mathrm{~h}$ under closed conditions and then cooled to $0^{\circ} \mathrm{C}$ for $30 \mathrm{~min}$. The generated $\mathrm{H}_{2}$ was released carefully in a fumehood. $n$-Hexane $(40 \mathrm{~mL})$ was then added to the reaction mixture. The resulting solid was collected and further washed with cold diethyl ether to afford the corresponding product.

\section{Characterization Data}

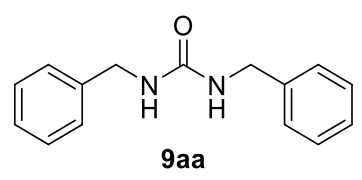

\section{1,3-dibenzylurea}

White solid (223.9 mg $0.933 \mathrm{mmol}, 93 \%)$. The compound was identified by spectral comparison with literature data. ${ }^{3}$

${ }^{1} \mathrm{H}$ NMR (300 MHz, DMSO-d $\left.6, \delta\right): 4.23\left(\mathrm{~s}, 4 \mathrm{H}, 2 \mathrm{CH}_{2}\right), 6.45(\mathrm{br}, 2 \mathrm{H}, 2 \mathrm{NH}), 7.20-7.34(\mathrm{~m}, 10 \mathrm{H}$, 10CH) ppm.

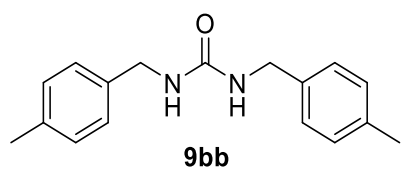

\section{1,3-bis(4-methylbenzyl)urea}

White solid (239.9 mg, $0.895 \mathrm{mmol}, 90 \%)$. The compound was identified by spectral comparison 
with literature data. ${ }^{4}$

${ }^{1} \mathrm{H}$ NMR (300 MHz, DMSO-d $\left.d_{6}, \delta\right): 2.28\left(\mathrm{~s}, 6 \mathrm{H}, 2 \mathrm{CH}_{3}\right), 4.17\left(\mathrm{~d}, J=5.6 \mathrm{~Hz}, 4 \mathrm{H}, 2 \mathrm{CH}_{2}\right), 6.35$ (br, 2 $\mathrm{H}, 2 \mathrm{NH}), 7.13(\mathrm{~m}, 8 \mathrm{H}, 8 \mathrm{CH}) \mathrm{ppm}$.

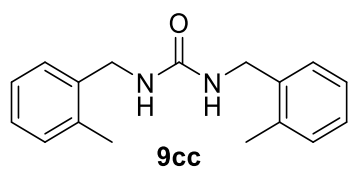

\section{1,3-bis(2-methylbenzyl)urea}

White solid (246.1 mg, $0.917 \mathrm{mmol}, 92 \%) .{ }^{1} \mathrm{H}$ NMR (300 MHz, DMSO-d,$\delta$ ): 2.27 (s, 6H, 2CH 3 ), $4.21\left(\mathrm{~d}, J=3 \mathrm{~Hz}, 4 \mathrm{H}, 2 \mathrm{CH}_{2}\right), 6.28(\mathrm{br}, 2 \mathrm{H}, 2 \mathrm{NH}), 7.15-7.23(\mathrm{~m}, 8 \mathrm{H}, 8 \mathrm{CH}) \mathrm{ppm} ;{ }^{13} \mathrm{C} \mathrm{NMR}(75$ MHz, DMSO- $\left.d_{6}, \delta\right): 18.98,41.53,126.16,127.10,127.63,130.32,135.84,138.85,158.33$ ppm, HRMS(ESI) calcd for $\mathrm{C}_{17} \mathrm{H}_{20} \mathrm{~N}_{2} \mathrm{ONa}$ : 291.1473 Found: 291.1468 [M+Na] .

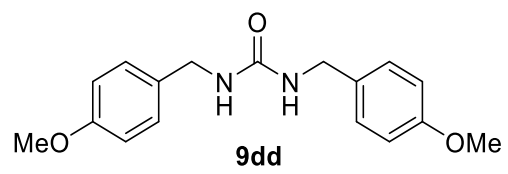

\section{1,3-bis(4-methoxybenzyl)urea}

White solid (226.0 mg, $0.753 \mathrm{mmol}, 75 \%$ ). The compound was identified by spectral comparison with literature data. ${ }^{5}$

${ }^{1} \mathrm{H}$ NMR $\left(300 \mathrm{MHz}, \mathrm{DMSO}-d_{6}, \delta\right): 3.73\left(\mathrm{~s}, 6 \mathrm{H}, 2 \mathrm{CH}_{3}\right), 4.14\left(\mathrm{~d}, J=6.0 \mathrm{~Hz}, 4 \mathrm{H}, 2 \mathrm{CH}_{2}\right), 6.29(\mathrm{~m}, 2 \mathrm{H}$, 2NH), $6.80(\mathrm{~d}, J=8.4 \mathrm{~Hz}, 4 \mathrm{H}, 4 \mathrm{CH}), 7.16(\mathrm{~d}, J=8.5 \mathrm{~Hz}, 4 \mathrm{H}, 4 \mathrm{CH}) \mathrm{ppm}$.

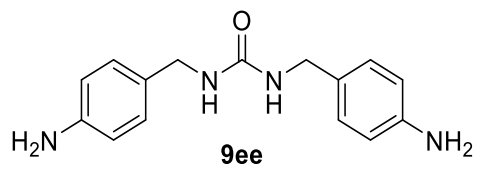

\section{1,3-bis(4-aminobenzyl)urea}

Reddish brown solid (227.1 $\mathrm{mg}, 0.841 \mathrm{mmol}, 84 \%)$. The compound was identified by spectral comparison with literature data. ${ }^{6}$ 
${ }^{1} \mathrm{H}$ NMR (300 MHz, DMSO- $\left.d_{6}, \delta\right): 4.01\left(\mathrm{~d}, J=5.5 \mathrm{~Hz}, 4 \mathrm{H}, 2 \mathrm{CH}_{2}\right), 4.93\left(\mathrm{~s}, 4 \mathrm{H}, 2 \mathrm{NH}_{2}\right), 6.04(\mathrm{br}, 2 \mathrm{H}$, 2NH), $6.49(\mathrm{~d}, J=8.3 \mathrm{~Hz}, 4 \mathrm{H}, 4 \mathrm{CH}), 6.89(\mathrm{~d}, J=8.3 \mathrm{~Hz}, 4 \mathrm{H}, 4 \mathrm{CH}) \mathrm{ppm}$.

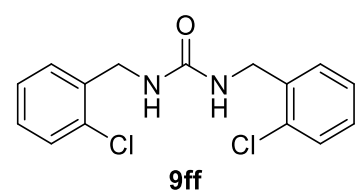

\section{1,3-bis(2-chlorobenzyl)urea}

White solid (232.1 mg, $0.751 \mathrm{mmol}, 75 \%) .{ }^{1} \mathrm{H}$ NMR (300 MHz, DMSO- $\left.d_{6}, \delta\right): 4.28$ (d, $J=5.9 \mathrm{~Hz}$, $\left.4 \mathrm{H}, 2 \mathrm{CH}_{2}\right), 7.07$ (br, 2H, 2NH), $7.28-7.43(\mathrm{~m}, 8 \mathrm{H}, 8 \mathrm{CH}) \mathrm{ppm} ;{ }^{13} \mathrm{C}$ NMR (75 MHz, DMSO- $\left.d_{6}, \delta\right)$ : 41.28, 127.54, 128.76, 129.03, 129.43, 132.26, 138.37, $158.53 \mathrm{ppm}$; HRMS(ESI) calcd for $\mathrm{C}_{15} \mathrm{H}_{14} \mathrm{Cl}_{2} \mathrm{~N}_{2} \mathrm{ONa}$ : 331.0381 Found: $331.0375[\mathrm{M}+\mathrm{Na}]^{+}$.

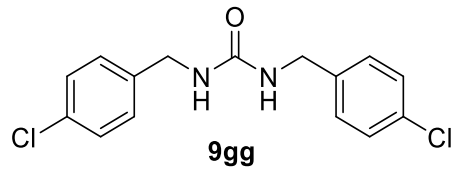

\section{1,3-bis(4-chlorobenzyl)urea}

White solid (216.3 $\mathrm{mg}, 0.700 \mathrm{mmol}, 70 \%)$. The compound was identified by spectral comparison with literature data. ${ }^{6}$

${ }^{1} \mathrm{H}$ NMR $\left(300 \mathrm{MHz}, \mathrm{DMSO}-d_{6}, \delta\right): 4.20\left(\mathrm{~d}, J=6.1 \mathrm{~Hz}, 4 \mathrm{H}, 2 \mathrm{CH}_{2}\right), 6.68(\mathrm{br}, 2 \mathrm{H}, 2 \mathrm{NH}), 7.25(\mathrm{~d}, J=$ $8.4 \mathrm{~Hz}, 4 \mathrm{H}, 4 \mathrm{CH}), 7.36$ (d, $J=8.4 \mathrm{~Hz}, 4 \mathrm{H}, 4 \mathrm{CH}) \mathrm{ppm}$.

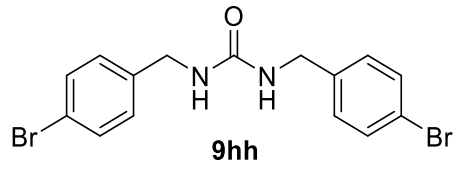

\section{1,3-bis(4-bromobenzyl)urea}

White solid (277.0 mg, $0.695 \mathrm{mmol}, 70 \%)$. The compound was identified by spectral comparison with literature data. ${ }^{6}$ 
${ }^{1} \mathrm{H}$ NMR $\left(300 \mathrm{MHz}, \mathrm{DMSO}-d_{6}, \delta\right): 4.18\left(\mathrm{~d}, J=6.0 \mathrm{~Hz}, 4 \mathrm{H}, 2 \mathrm{CH}_{2}\right), 6.59(\mathrm{br}, 2 \mathrm{H}, 2 \mathrm{NH}), 7.19(\mathrm{~d}, J=$ $8.2 \mathrm{~Hz}, 4 \mathrm{H}, 4 \mathrm{CH}), 7.49$ (d, $J=8.3 \mathrm{~Hz}, 4 \mathrm{H}, 4 \mathrm{CH}) \mathrm{ppm}$.

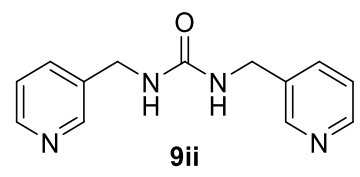

\section{1,3-bis(pyridin-3-ylmethyl)urea}

White solid (185.1 mg, $0.765 \mathrm{mmol}, 77 \%) .{ }^{1} \mathrm{H}$ NMR (300 MHz, DMSO- $\left.d_{6}, \delta\right): 4.24(\mathrm{~d}, J=6.0 \mathrm{~Hz}$, $\left.4 \mathrm{H}, 2 \mathrm{CH}_{2}\right), 6.62(\mathrm{br}, 2 \mathrm{H}, 2 \mathrm{NH}), 7.31(\mathrm{~m}, 2 \mathrm{H}, 2 \mathrm{CH}), 7.63(\mathrm{~d}, J=7.8 \mathrm{~Hz}, 2 \mathrm{H}, 2 \mathrm{CH}), 8.43(\mathrm{~m}, 4 \mathrm{H}$, 4CH) ppm; ${ }^{13} \mathrm{C}$ NMR (75 MHz, DMSO- $\left.d_{6}, \delta\right): 41.16,123.83,135.27,135.74,148.34,149.06,158.51$ ppm; HRMS(ESI) calcd for $\mathrm{C}_{13} \mathrm{H}_{14} \mathrm{~N}_{4} \mathrm{ONa}$ : 265.1065 Found: $265.1060[\mathrm{M}+\mathrm{Na}]^{+}$.

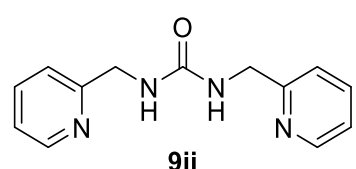

$9 \mathrm{jj}$

\section{1,3-bis(pyridin-2-ylmethyl)urea}

Yellowish solid (140.4 mg, $0.580 \mathrm{mmol}, 58 \%) .{ }^{1} \mathrm{H}$ NMR (300 MHz, DMSO- $d_{6}, \delta$ ): 4.33 (d, $J=5.8$ $\left.\mathrm{Hz}, 4 \mathrm{H}, 2 \mathrm{CH}_{2}\right), 6.74(\mathrm{~m}, 2 \mathrm{H}, 2 \mathrm{NH}), 7.23-7.32(\mathrm{~m}, 4 \mathrm{H}, 4 \mathrm{CH}), 7.73-7.79(\mathrm{~m}, 2 \mathrm{H}, 2 \mathrm{CH}), 8.49(\mathrm{~d}, J$ $=4.6 \mathrm{~Hz}, 2 \mathrm{H}, 2 \mathrm{CH}) \mathrm{ppm} ;{ }^{13} \mathrm{C} \mathrm{NMR}\left(75 \mathrm{MHz}, \mathrm{DMSO}-d_{6}, \delta\right): 45.42,121.30,122.36,137.08,149.16$, 158.59, 160.18 ppm; HRMS(ESI) calcd for $\mathrm{C}_{13} \mathrm{H}_{14} \mathrm{~N}_{4} \mathrm{ONa}$ : 265.1065 Found: 265.1060 [M+Na $]^{+}$.

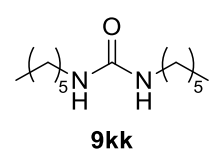

\section{1,3-di-n-hexylurea}

Purification of the product was performed with silica gel column chromatography using DCM/methanol (20:1) as an eluent. Thick dark oil (199.1 mg, $0.873 \mathrm{mmol}, 87 \%)$. The compound was identified by spectral comparison with literature data. ${ }^{3}$

${ }^{1} \mathrm{H} \mathrm{NMR}\left(300 \mathrm{MHz}, \mathrm{CDCl}_{3}, \delta\right): 0.88\left(\mathrm{t}, J=6.6 \mathrm{~Hz}, 6 \mathrm{H}, 2 \mathrm{CH}_{3}\right), 1.29\left(\mathrm{~m}, 12 \mathrm{H}, 6 \mathrm{CH}_{2}\right), 1.48(\mathrm{~m}, 4 \mathrm{H}$, S6 
$\left.2 \mathrm{CH}_{2}\right), 3.15$ (br, $\left.4 \mathrm{H}, 2 \mathrm{CH}_{2}\right), 4.30(\mathrm{br}, 2 \mathrm{H}, 2 \mathrm{NH}) \mathrm{ppm}$.

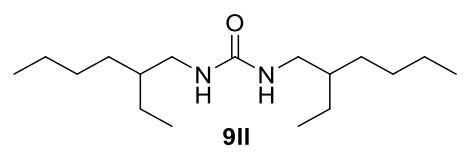

\section{1,3-bis(2-ethylhexyl)urea}

Purification of the product was performed with silica gel column chromatography using DCM/methanol (20:1) as an eluent. Thick dark oil (270.1 mg, $0.951 \mathrm{mmol}, 95 \%)$.

${ }^{1} \mathrm{H}$ NMR $\left(300 \mathrm{MHz}, \mathrm{CDCl}_{3}, \delta\right): 0.89\left(\mathrm{t}, J=7.6 \mathrm{~Hz}, 12 \mathrm{H}, 4 \mathrm{CH}_{3}\right), 1.28-1.41(\mathrm{~m}, 18 \mathrm{H}), 3.09(\mathrm{~d}, J=$

$\left.5.9 \mathrm{~Hz}, 4 \mathrm{H}, 2 \mathrm{CH}_{2}\right), 4.25$ (br, 2H, 2NH) ppm; ${ }^{13} \mathrm{C} \mathrm{NMR}\left(75 \mathrm{MHz}, \mathrm{CDCl}_{3}, \delta\right): 10.92,14.07,23.05$, 24.22, 28.94, 31.01, 39.81, 43.54, 158.44 ppm; HRMS(ESI) calcd for $\mathrm{C}_{17} \mathrm{H}_{36} \mathrm{~N}_{2} \mathrm{ONa}$ : 307.2725 Found: $307.2720[\mathrm{M}+\mathrm{Na}]^{+}$.

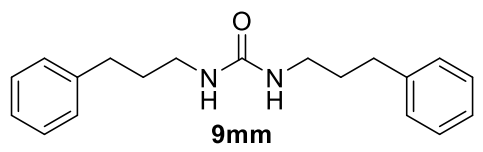

\section{1,3-bis(3-phenylpropyl)urea}

White solid (267.0 mg, $0.902 \mathrm{mmol}, 90 \%) .{ }^{1} \mathrm{H}$ NMR (300 MHz, DMSO- $\left.d_{6}, \delta\right): 1.66$ (m, 4H, 2CH 2 ), $2.57\left(\mathrm{~m}, 4 \mathrm{H}, 2 \mathrm{CH}_{2}\right), 2.99\left(\mathrm{~m}, 4 \mathrm{H}, 2 \mathrm{CH}_{2}\right), 5.86(\mathrm{~m}, 2 \mathrm{H}, 2 \mathrm{NH}), 7.15-7.30(\mathrm{~m}, 10 \mathrm{H}, 10 \mathrm{CH}) \mathrm{ppm} ;{ }^{13} \mathrm{C}$ NMR (75 MHz, DMSO-d $\left.d_{6}, \delta\right): 32.34,32.97,39.33,126.13,128.73,142.32,158.57$ ppm; HRMS(ESI) calcd for $\mathrm{C}_{19} \mathrm{H}_{24} \mathrm{~N}_{2} \mathrm{ONa}$ : 319.1786 Found: $319.1781[\mathrm{M}+\mathrm{Na}]^{+}$.

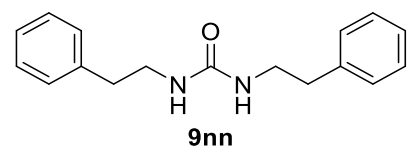

\section{1,3-bis(phenethyl)urea}

White solid (252.0 mg, $0.942 \mathrm{mmol}, 94 \%)$. The compound was identified by spectral comparison with literature data. ${ }^{7}$

${ }^{1} \mathrm{H}$ NMR (300 MHz, DMSO- $\left.d_{6}, \delta\right): 2.64\left(\mathrm{t}, J=7.2 \mathrm{~Hz}, 4 \mathrm{H}, 2 \mathrm{CH}_{2}\right), 3.20(\mathrm{dd}, J=6.7 \mathrm{~Hz}, J=13.3 \mathrm{~Hz}$, 
$\left.4 \mathrm{H}, 2 \mathrm{CH}_{2}\right), 5.88(\mathrm{~m}, 2 \mathrm{H}, 2 \mathrm{NH}), 7.13-7.31(\mathrm{~m}, 10 \mathrm{H}, 10 \mathrm{CH}) \mathrm{ppm}$.

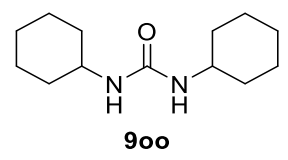

\section{1,3-dicyclohexylurea}

White solid (182.3 mg, $0.813 \mathrm{mmol}, 81 \%)$. The compound was identified by spectral comparison with literature data. ${ }^{8}$

${ }^{1} \mathrm{H}$ NMR $\left(300 \mathrm{MHz}, \mathrm{MeOD}-d_{4}, \delta\right): 1.09-1.43(\mathrm{~m}, 10 \mathrm{H}), 1.59-1.88(\mathrm{~m}, 10 \mathrm{H}), 3.31-3.50(\mathrm{~m}, 2 \mathrm{H}$, 2CH) ppm.

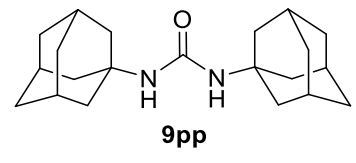

\section{1,3-di(1-adamantyl)urea}

White solid (103.1 mg, $0.314 \mathrm{mmol}, 31 \%$ ), The compound was identified by spectral comparison with literature data. ${ }^{9}$

${ }^{1} \mathrm{H}$ NMR $\left(300 \mathrm{MHz}, \mathrm{CDCl}_{3}, \delta\right): 1.66(\mathrm{~m}, 12 \mathrm{H}), 1.95(\mathrm{~m}, 12 \mathrm{H}), 2.05(\mathrm{~m}, 6 \mathrm{H}), 3.83(\mathrm{br}, 2 \mathrm{H}, 2 \mathrm{NH}) \mathrm{ppm}$.<smiles>O=C1NC2CCCCC2N1</smiles>

\section{Octahydro-benzoimidazol-2-one}

Purification of the product was performed with silica gel column chromatography using DCM/methanol (20:1) as an eluent. White solid (105 $\mathrm{mg}, 0.751 \mathrm{mmol}, 75 \%)$. The compound was identified by spectral comparison with literature data. ${ }^{8}$

${ }^{1} \mathrm{H}$ NMR (300 MHz, MeOD-d4, $\left.\delta\right): 1.36(\mathrm{~m}, 2 \mathrm{H}), 1.56-1.75(\mathrm{~m}, 6 \mathrm{H}), 3.67(\mathrm{~m}, 2 \mathrm{H}, 2 \mathrm{CH}) \mathrm{ppm}$. 


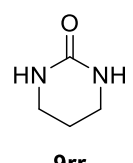

\section{Tetrahydropyrimidin-2(1H)-one}

White solid (51.1mg, $0.511 \mathrm{mmol}, 51 \%) .{ }^{1} \mathrm{H}$ NMR (300 MHz, $\left.\mathrm{D}_{2} \mathrm{O}, \delta\right): 1.85(\mathrm{~m}, 2 \mathrm{H}), 3.26(\mathrm{~m}, 4 \mathrm{H})$ ppm.

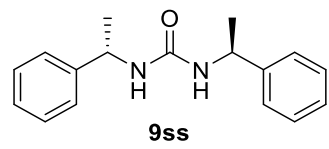

\section{1,3-bis((S)-1-phenylethyl)urea}

White solid (164.2 mg, $0.611 \mathrm{mmol}, 61 \%)$. The compound was identified by spectral comparison with literature data. ${ }^{7}$

${ }^{1} \mathrm{H}$ NMR $\left(300 \mathrm{MHz}, \mathrm{DMSO}-d_{6}, \delta\right): 1.28\left(\mathrm{~s}, J=6.9 \mathrm{~Hz}, 6 \mathrm{H}, 2 \mathrm{CH}_{3}\right), 4.72(\mathrm{~m}, 2 \mathrm{H}, 2 \mathrm{CH}), 6.26(\mathrm{~d}, J=$ $8.0 \mathrm{~Hz}, 2 \mathrm{H}, 2 \mathrm{NH}), 7.19-7.34(\mathrm{~m}, 10 \mathrm{H}, 10 \mathrm{CH}) \mathrm{ppm}$.

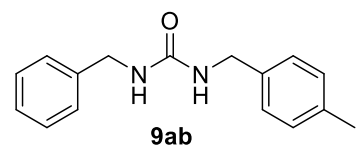

\section{1-benzyl-3-(4-methylbenzyl)urea}

Yellowish solid (94.9 mg, $0.373 \mathrm{mmol}, 89 \%)$. The compound was identified by spectral comparison with literature data. ${ }^{10}$

${ }^{1} \mathrm{H}$ NMR (300 MHz, $\mathrm{CDCl}_{3}, \delta$ ): $2.31\left(\mathrm{~s}, 3 \mathrm{H}, \mathrm{CH}_{3}\right), 4.32\left(\mathrm{~d}, J=6.0,2 \mathrm{H}, \mathrm{CH}_{2}\right), 4.37(\mathrm{~d}, J=5.8,2 \mathrm{H}$, $\left.\mathrm{CH}_{2}\right), 4.80$ (br, 2H, 2NH), $7.15-7.09(\mathrm{~m}, 4 \mathrm{H}, 4 \mathrm{CH}), 7.25-7.32(\mathrm{~m}, 5 \mathrm{H}, 5 \mathrm{CH}) \mathrm{ppm}$.

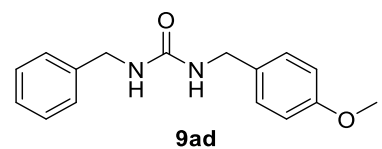

\section{1-benzyl-3-(4-methoxybenzyl)urea}

Yellowish solid (101.3 mg, $0.375 \mathrm{mmol}, 90 \%)$. The compound was identified by spectral comparison 
with literature data. ${ }^{10}$

${ }^{1} \mathrm{H}$ NMR (300 MHz, $\mathrm{CDCl}_{3}, \delta$ ): 3.77 (s, 3H, $\left.\mathrm{CH}_{3}\right), 4.29$ (m, 2H, $\left.\mathrm{CH}_{2}\right), 4.36\left(\mathrm{~m}, 2 \mathrm{H}, \mathrm{CH}_{2}\right), 4.71$ (br, $2 \mathrm{H}, 2 \mathrm{NH}), 6.82(\mathrm{~d}, J=8.0,2 \mathrm{H}, 2 \mathrm{CH}), 7.18(\mathrm{~d}, J=8.0,2 \mathrm{H}, 2 \mathrm{CH}), 7.25-7.30(\mathrm{~m}, 5 \mathrm{H}, 5 \mathrm{CH}) \mathrm{ppm}$.

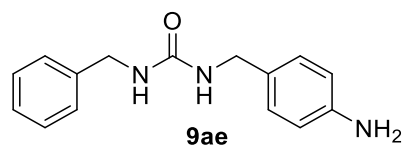

\section{1-(4-aminobenzyl)-3-benzylurea}

Light orange solid (94.2 mg, $0.369 \mathrm{mmol}, 88 \%) .{ }^{1} \mathrm{H}$ NMR (300 MHz, DMSO- $\left.d_{6}, \delta\right): 4.03(\mathrm{~d}, J=5.7$ $\left.\mathrm{Hz}, 2 \mathrm{H}, \mathrm{CH}_{2}\right), 4.21\left(\mathrm{~d}, J=5.9,2 \mathrm{H}, \mathrm{CH}_{2}\right), 4.92\left(\mathrm{~s}, 2 \mathrm{H}, \mathrm{NH}_{2}\right), 6.20(\mathrm{~m}, 1 \mathrm{H}, \mathrm{NH}), 6.35(\mathrm{~m}, 1 \mathrm{H}, \mathrm{NH})$, $6.49(\mathrm{~d}, J=8.3 \mathrm{~Hz}, 2 \mathrm{H}, 2 \mathrm{CH}), 6.90(\mathrm{~d}, J=8.2 \mathrm{~Hz}, 2 \mathrm{H}, 2 \mathrm{CH}), 7.20-7.34(\mathrm{~m}, 5 \mathrm{H}, 5 \mathrm{CH}) \mathrm{ppm} ;{ }^{13} \mathrm{C}$ NMR (75 MHz, DMSO- $\left.d_{6}, \delta\right): 43.30,43.38,114.15,126.98,127.44,127.98,128.54,128.65,141.44$, 147.85, 158.49 ppm; HRMS(ESI) calcd for $\mathrm{C}_{15} \mathrm{H}_{17} \mathrm{~N}_{3} \mathrm{ONa}$ : 278.1269 Found: 278.1264 [M+Na] ${ }^{+}$.

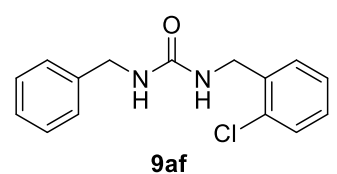

\section{1-benzyl-3-(2-chlorobenzyl)urea}

White solid (67.4 mg, $0.246 \mathrm{mmol}, 59 \%)$. The compound was identified by spectral comparison with literature data. ${ }^{10}$

${ }^{1} \mathrm{H}$ NMR (300 MHz, $\mathrm{CDCl}_{3}, \delta$ ): 4.37 (br, 2H, $\mathrm{CH}_{2}$ ), 4.46 (br, 2H, $\mathrm{CH}_{2}$ ), 4.80 (br, 1H, NH), 4.93 (br, 1H, NH), $7.17-7.40(\mathrm{~m}, 9 \mathrm{H}, 9 \mathrm{CH}) \mathrm{ppm}$.

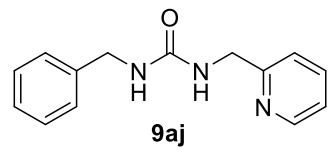

\section{1-benzyl-3-(pyridin-2-ylmethyl)urea}

White solid ( $84.1 \mathrm{mg}, 0.349 \mathrm{mmol}, 83 \%)$. The compound was identified by spectral comparison with 
literature data. ${ }^{10}$

${ }^{1} \mathrm{H}$ NMR (300 MHz, $\mathrm{CDCl}_{3}, \delta$ ): 4.41 (d, $\left.J=5.8,2 \mathrm{H}, \mathrm{CH}_{2}\right), 4.50\left(\mathrm{~d}, J=5.8,2 \mathrm{H}, \mathrm{CH}_{2}\right), 5.22$ (br, 1H, NH), 5.67 (br, 1H, NH), $7.16-7.18(\mathrm{~m}, 1 \mathrm{H}, 1 \mathrm{CH}), 7.28-7.36(\mathrm{~m}, 6 \mathrm{H}, 6 \mathrm{CH}), 7.64-7.69(\mathrm{~m}, 1 \mathrm{H})$, $8.48(\mathrm{~d}, J=4.6,1 \mathrm{H}) \mathrm{ppm}$.

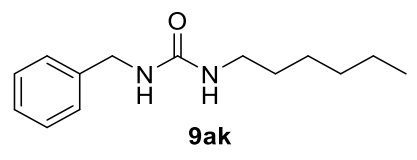

\section{1-benzyl-3-n-hexylurea}

White solid (77.7 mg, $0.332 \mathrm{mmol}, 79 \%) .{ }^{1} \mathrm{H}$ NMR (300 MHz, DMSO- $\left.d_{6}, \delta\right): 0.87\left(\mathrm{~m}, 3 \mathrm{H}, \mathrm{CH}_{3}\right)$, $1.26-1.37\left(\mathrm{~m}, 8 \mathrm{H}, 4 \mathrm{CH}_{2}\right), 2.97\left(\mathrm{~m}, 2 \mathrm{H}, \mathrm{CH}_{2}\right), 4.19\left(\mathrm{~m}, 2 \mathrm{H}, \mathrm{CH}_{2}\right), 5.91(\mathrm{~m}, 1 \mathrm{H}, \mathrm{NH}), 6.27(\mathrm{~m}, 1 \mathrm{H}$, $\mathrm{NH}), 7.21-7.34(\mathrm{~m}, 5 \mathrm{H}, 5 \mathrm{CH}) \mathrm{ppm} ;{ }^{13} \mathrm{C} \mathrm{NMR}\left(75 \mathrm{MHz}, \mathrm{DMSO}-d_{6}, \delta\right): 14.38,22.57,26.51,30.46$, $31.51,39.78,43.33,126.94,127.42,126.62,141.52,158.53$ ppm; HRMS(ESI) calcd for $\mathrm{C}_{14} \mathrm{H}_{22} \mathrm{~N}_{2} \mathrm{ONa}$ : 257.1630 Found: $257.1624[\mathrm{M}+\mathrm{Na}]^{+}$.

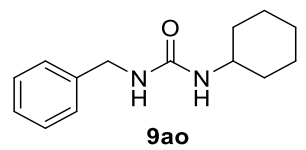

\section{1-benzyl-3-cyclohexylurea}

White solid ( $80.8 \mathrm{mg}, 0.348 \mathrm{mmol}, 83 \%)$. The compound was identified by spectral comparison with literature data. $^{4}$

${ }^{1} \mathrm{H}$ NMR (300 MHz, DMSO- $\left.d_{6}, \delta\right): 1.03-1.29(\mathrm{~m}, 5 \mathrm{H}), 1.50-1.77(\mathrm{~m}, 5 \mathrm{H}), 3.39(\mathrm{~m}, 1 \mathrm{H}, \mathrm{CH}), 4.18$ $\left(\mathrm{d}, J=5.9,2 \mathrm{H}, \mathrm{CH}_{2}\right), 5.81(\mathrm{~d}, J=7.9,1 \mathrm{H}, \mathrm{NH}), 6.17(\mathrm{~m}, 1 \mathrm{H}, \mathrm{NH}), 7.22-7.33(\mathrm{~m}, 5 \mathrm{H}, 5 \mathrm{CH}) \mathrm{ppm}$.

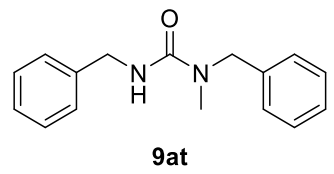

1,3-dibenzyl-1-methylurea 
Purification of the product was performed with silica gel column chromatography using EA $/ n$-hexane (1:1) as an eluent. White solid (74.0 $\mathrm{mg}, 0.291 \mathrm{mmol}, 70 \%)$. The compound was identified by spectral comparison with literature data. ${ }^{10}$

${ }^{1} \mathrm{H}$ NMR (300 MHz, $\mathrm{CDCl}_{3}, \delta$ ): $2.92\left(\mathrm{~s}, 3 \mathrm{H}, \mathrm{CH}_{3}\right), 4.47$ (d, J = 5.5 Hz, 2H, $\left.\mathrm{CH}_{2}\right), 4.55\left(\mathrm{~s}, 2 \mathrm{H}, \mathrm{CH}_{2}\right)$, 4.74 (br, 1H, NH), $7.26-7.38(\mathrm{~m}, 10 \mathrm{H}, 10 \mathrm{CH}) \mathrm{ppm}$.

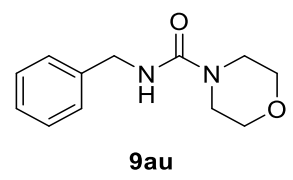

\section{N-benzylmorpholine-4-carboxamide}

Purification of the product was performed with silica gel column chromatography using DCM/methanol (20:1) as an eluent. White solid (68.4 mg, $0.311 \mathrm{mmol}, 74 \%)$. The compound was identified by spectral comparison with literature data. ${ }^{10}$

${ }^{1} \mathrm{H}$ NMR (300 MHz, $\left.\mathrm{CDCl}_{3}, \delta\right): 3.37\left(\mathrm{t}, J=4.8 \mathrm{~Hz}, 4 \mathrm{H}, 2 \mathrm{CH}_{2}\right), 3.70\left(\mathrm{t}, J=5.1 \mathrm{~Hz}, 4 \mathrm{H}, 2 \mathrm{CH}_{2}\right), 4.44$ $\left(\mathrm{d}, J=5.5 \mathrm{~Hz}, 2 \mathrm{H}, \mathrm{CH}_{2}\right), 4,79(\mathrm{br}, 1 \mathrm{H}, \mathrm{NH}), 7.27-7.39(\mathrm{~m}, 5 \mathrm{H}, 5 \mathrm{CH}) \mathrm{ppm}$.

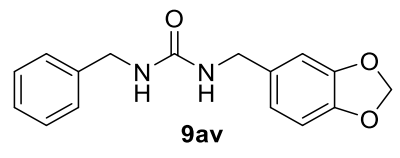

\section{1-(benzo-1,3-dioxol-5-ylmethyl)-3-benzylurea}

White solid (99.1 mg, $0.348 \mathrm{mmol}, 83 \%) .{ }^{1} \mathrm{H}$ NMR (300 MHz, DMSO-d6, $\delta$ ): 4.12 (d, $J=6.0 \mathrm{~Hz}$, $\left.2 \mathrm{H}, \mathrm{CH}_{2}\right), 4.22\left(\mathrm{~d}, J=6.0 \mathrm{~Hz}, 2 \mathrm{H}, \mathrm{CH}_{2}\right), 5.98\left(\mathrm{~s}, 2 \mathrm{H}, \mathrm{CH}_{2}\right), 6.36-6.44(\mathrm{~m}, 2 \mathrm{H}, 2 \mathrm{NH}), 6.71-6.74$ (m, 1H, CH), $6.81-6.83(\mathrm{~m}, 2 \mathrm{H}, 2 \mathrm{CH}), 7.22-7.34(\mathrm{~m}, 5 \mathrm{H}, 5 \mathrm{CH}) \mathrm{ppm} ;{ }^{13} \mathrm{C}$ NMR $(75 \mathrm{MHz}, \mathrm{DMSO}-$ $\left.d_{6}, \delta\right): 43.22,43.40,101.19,108.14,108.40,120.54,127.00,127.44,126.66,135.34,141.36,146.31$, 147.64, 158.48 ppm; HRMS(ESI) calcd for $\mathrm{C}_{16} \mathrm{H}_{16} \mathrm{~N}_{2} \mathrm{O}_{3} \mathrm{Na}$ : 307.1059 Found: 307.1053 [M+Na] $]^{+}$ 


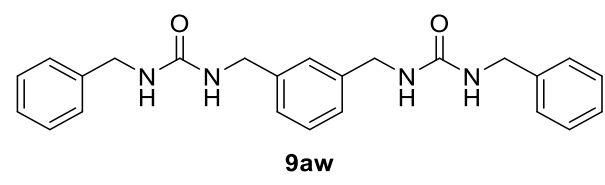

\section{1,1'-(1,3-phenylenebis(methylene))bis(3-benzylurea)}

White solid (75.1 mg, $0.187 \mathrm{mmol}, 89 \%) .{ }^{1} \mathrm{H} \mathrm{NMR}\left(300 \mathrm{MHz}, \mathrm{DMSO}-d_{6}, \delta\right): 4.23(\mathrm{t}, J=5.9 \mathrm{~Hz}, 8 \mathrm{H}$, $\left.4 \mathrm{CH}_{2}\right), 6.44(\mathrm{~m}, 4 \mathrm{H}, 4 \mathrm{NH}), 7.11-7.35(\mathrm{~m}, 14 \mathrm{H}, 14 \mathrm{CH}) \mathrm{ppm} ;{ }^{13} \mathrm{C}$ NMR $\left(75 \mathrm{MHz}, \mathrm{DMSO}-d_{6}, \delta\right):$ $43.44,43.49,125.78,126.21,127.01,127.46,128.63,128.68,141.29,141.38,158.52 \mathrm{ppm}$; HRMS(ESI) calcd for $\mathrm{C}_{24} \mathrm{H}_{26} \mathrm{~N}_{4} \mathrm{O}_{2} \mathrm{Na}$ : 425.1953 Found: 425.1948 [M+Na] ${ }^{+}$. 


\section{Complementary Reaction Optimization Data}

Table S1. Screening Experiment for Symmetrical Urea Synthesis ${ }^{a}$

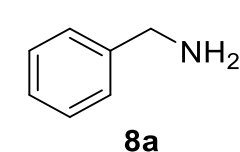

$8 \mathbf{a}$

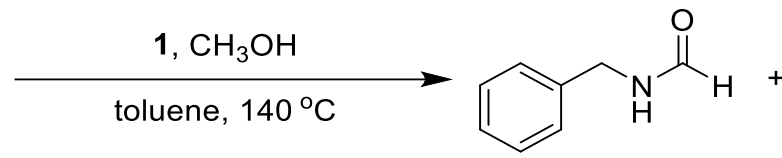

10<smiles>O=C(NCc1ccccc1)NCc1ccccc1</smiles>

9aa

\begin{tabular}{ccccccc}
\hline entry & loading & toluene & time & $\begin{array}{c}\text { MeOH } \\
\text { mmol }\end{array}$ & $\mathbf{1 0}(\%)$ & 9aa (\%) \\
\hline 1 & $0.5 \mathrm{~mol} \mathrm{\%}$ & $1.5 \mathrm{~mL}$ & $24 \mathrm{~h}$ & 20 & 30 & 52 \\
2 & $0.5 \mathrm{~mol} \%$ & $1.5 \mathrm{~mL}$ & $24 \mathrm{~h}$ & 10 & 17 & 82 \\
3 & $0.5 \mathrm{~mol} \%$ & $4.0 \mathrm{~mL}$ & $24 \mathrm{~h}$ & 7.5 & 20 & 68 \\
4 & $0.5 \mathrm{~mol} \%$ & $1.5 \mathrm{~mL}$ & $24 \mathrm{~h}$ & 7.5 & 14 & 84 \\
5 & $0.5 \mathrm{~mol} \%$ & $1.0 \mathrm{~mL}$ & $24 \mathrm{~h}$ & 7.5 & 12 & 80 \\
6 & $0.5 \mathrm{~mol} \%$ & $1.5 \mathrm{~mL}$ & $12 \mathrm{~h}$ & 7.5 & 22 & 63 \\
7 & $0.5 \mathrm{~mol} \%$ & $1.5 \mathrm{~mL}$ & $36 \mathrm{~h}$ & 7.5 & 15 & 83 \\
8 & $0.5 \mathrm{~mol} \%$ & $1.5 \mathrm{~mL}$ & $24 \mathrm{~h}$ & 4.0 & 10 & 88 \\
9 & $0.5 \mathrm{~mol} \%$ & $1.5 \mathrm{~mL}$ & $24 \mathrm{~h}$ & 2.0 & trace & 97 \\
$10^{b}$ & $0.5 \mathrm{~mol} \%$ & $1.5 \mathrm{~mL}$ & $24 \mathrm{~h}$ & 2.0 & 5 & 91 \\
$11^{c}$ & $0.5 \mathrm{~mol} \%$ & $1.5 \mathrm{~mL}$ & $24 \mathrm{~h}$ & 2.0 & 11 & 31 \\
12 & $0.5 \mathrm{~mol} \%$ & $1.5 \mathrm{~mL}$ & $24 \mathrm{~h}$ & 1.4 & 0 & 89
\end{tabular}

${ }^{a}$ Reaction conditions: benzyl amine (2.0 mmol, 1.0 equiv), 1, methanol, toluene, $140{ }^{\circ} \mathrm{C}$, NMR yield, $p$-xylene was used as a standard. ${ }^{b} 120{ }^{\circ} \mathrm{C} .{ }^{c} 100{ }^{\circ} \mathrm{C}$. 
Table S2. Turnover Numbers and Turnover Frequencies Measurement ${ }^{a}$<smiles>NCc1ccccc1</smiles>

$8 \mathbf{a}$<smiles>[CH2+]C(=O)NCc1ccccc1</smiles>

10<smiles>O=C(NCc1ccccc1)NCc1ccccc1</smiles>

9aa

\begin{tabular}{ccccccc}
\hline entry & loading & toluene & $\mathbf{1 0}(\%)$ & 9aa $(\%)$ & TON & TOF $(1 / \mathrm{h})$ \\
\hline 1 & $0.5 \mathrm{~mol} \mathrm{\%}$ & $1 \mathrm{~h}$ & 2 & 12 & 12 & 12 \\
2 & $0.5 \mathrm{~mol} \mathrm{\%}$ & $3 \mathrm{~h}$ & 2 & 32 & 32 & 10.7 \\
3 & $0.5 \mathrm{~mol} \mathrm{\%}$ & $6 \mathrm{~h}$ & 3 & 63 & 63 & 10.5 \\
4 & $0.5 \mathrm{~mol} \mathrm{\%}$ & $12 \mathrm{~h}$ & 4 & 78 & 78 & 6.5 \\
5 & $0.3 \mathrm{~mol} \mathrm{\%}$ & $12 \mathrm{~h}$ & 2 & 53 & 88 & 7.3 \\
6 & $0.5 \mathrm{~mol} \%$ & $24 \mathrm{~h}$ & trace & 97 & 97 & 4.0 \\
7 & $0.2 \mathrm{~mol} \%$ & $48 \mathrm{~h}$ & 4 & 85 & 213 & 4.4 \\
8 & $0.1 \mathrm{~mol} \%$ & $48 \mathrm{~h}$ & trace & 59 & 295 & 6.1
\end{tabular}

${ }^{a}$ Reaction conditions: benzyl amine (2.0 mmol, 1.0 equiv), 1 , methanol $(2.0 \mathrm{mmol})$, toluene, $140{ }^{\circ} \mathrm{C}$, NMR yield, $p$-xylene was used as a standard, TONs and TOFs were calculated based on the amount of 1,3-dibenzyl urea. 
Table S3. Screening Experiment for Unsymmetrical Urea Synthesis ${ }^{a}$

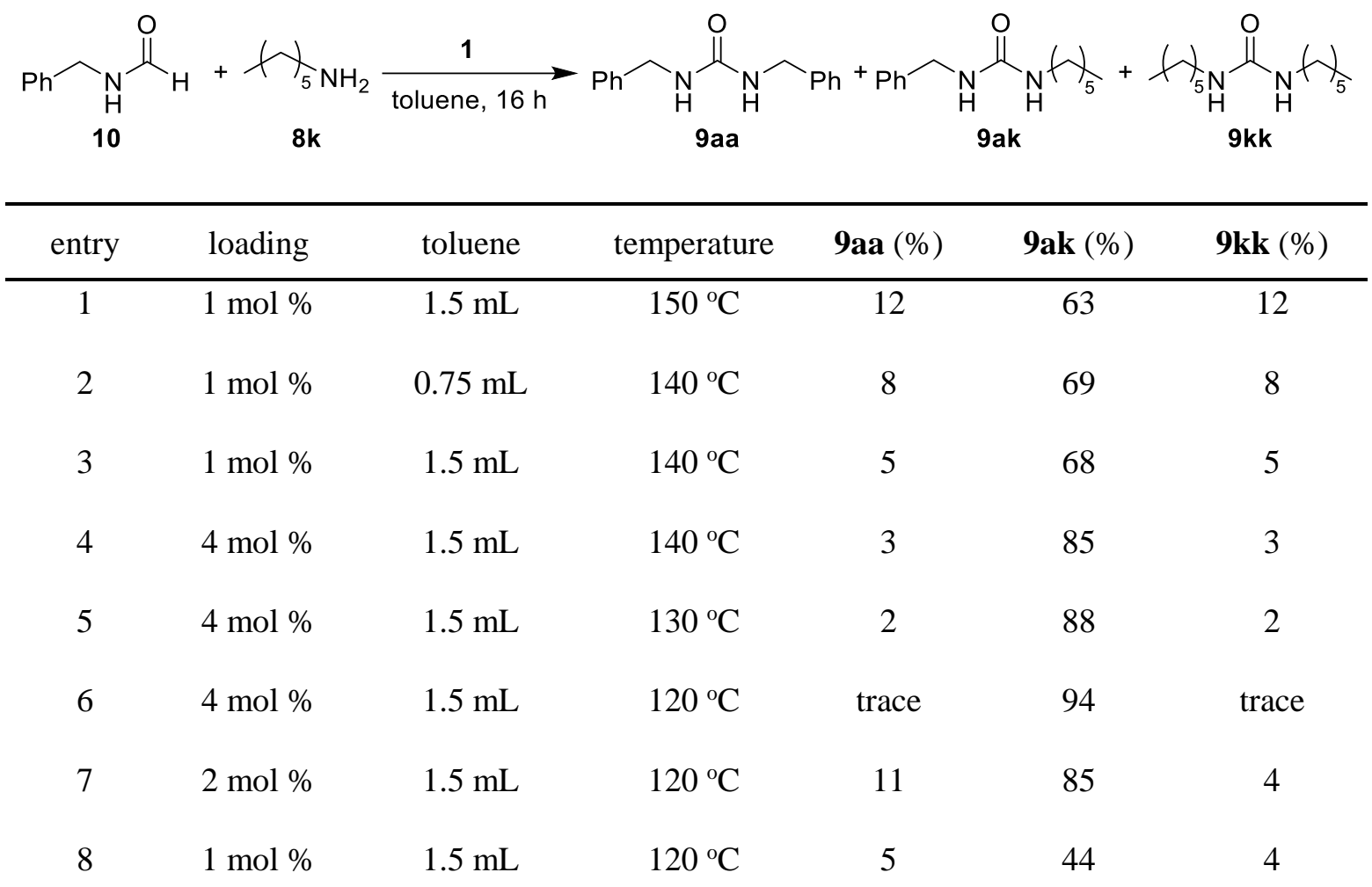

${ }^{a}$ Reaction conditions: 10 (0.5 mmol, 1.0 equiv), 8k (0.5 mmol), 1, toluene, NMR yield, $p$-xylene was used as a standard.

\section{Scheme S1. Attempts to Synthesize Unsymmetrical Urea Derivatives}

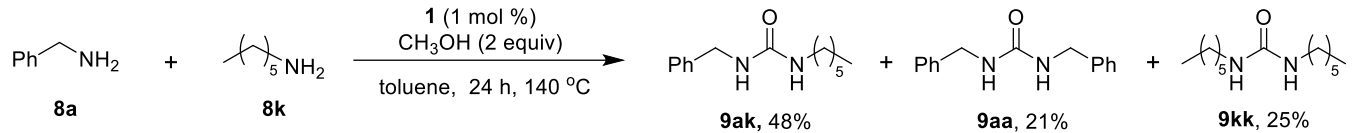

$$
\begin{aligned}
& \text { 8a }
\end{aligned}
$$

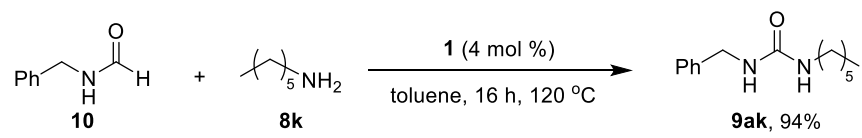




\section{Scheme S2. Screening Experiment for Unsymmetrical Urea Synthesis}

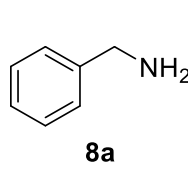

$0.5 \mathrm{mmol}$
1) $\mathrm{Ru}-\mathrm{MACHO}-\mathrm{BH}$

$\mathrm{MeOH}(2 \mathrm{~mL}), 12 \mathrm{~h}, 150^{\circ} \mathrm{C}$

2) $n$-hexylamine $(0.5 \mathrm{mmol})$

toluene $(1.5 \mathrm{~mL}), 16 \mathrm{~h}, 120^{\circ} \mathrm{C}$

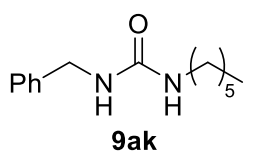

[Ru] $2 \mathrm{~mol} \%-48 \%$

[Ru] $4 \mathrm{~mol} \%-71 \%$

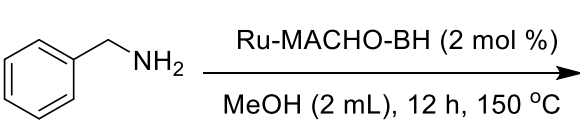

$8 a$<smiles>O=CNCc1ccccc1</smiles>

$75 \%$

\section{$0.50 \mathrm{mmol}, 1.2$ equiv}

$0.42 \mathrm{mmol}, 1.0$ equiv<smiles>O=C(NCc1ccccc1)NCc1ccccc1</smiles>

$\underset{\text { toluene }(1.5 \mathrm{~mL}), 16 \mathrm{~h}, 120^{\circ} \mathrm{C}}{\begin{array}{l}\text { Ru-MACHO-BH }(4 \mathrm{~mol} \%) \\ \text {-hexylamine }(0.42 \mathrm{mmol})\end{array}}$

$4 \%$

\section{Scheme S3. Control Experiments}

a)
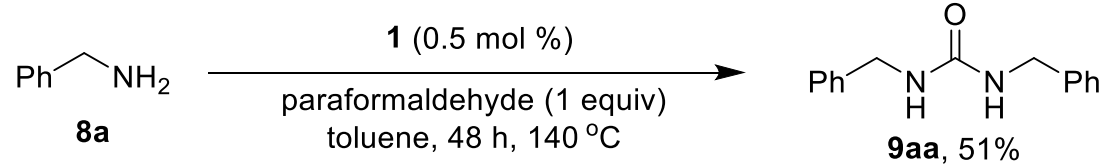

b)<smiles>O=CNCc1ccccc1</smiles>

10 $\underset{\text { toluene, } 120^{\circ} \mathrm{C}, 16 \mathrm{~h}}{\stackrel{1(4 \mathrm{~mol} \%)}{\longrightarrow}}$ no reaction $\left(\begin{array}{c}\mathrm{Ph} \\ \widehat{\sim}_{0 \%} \mathrm{NCO}\end{array}\right)$<smiles>O=CNCc1ccccc1</smiles>

10<smiles>Nc1ccccc1</smiles>

9x

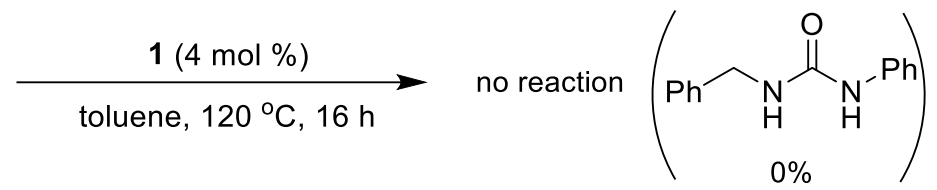


Figure S1. The LC chart for 1,3-bis(1-phenylethyl)urea

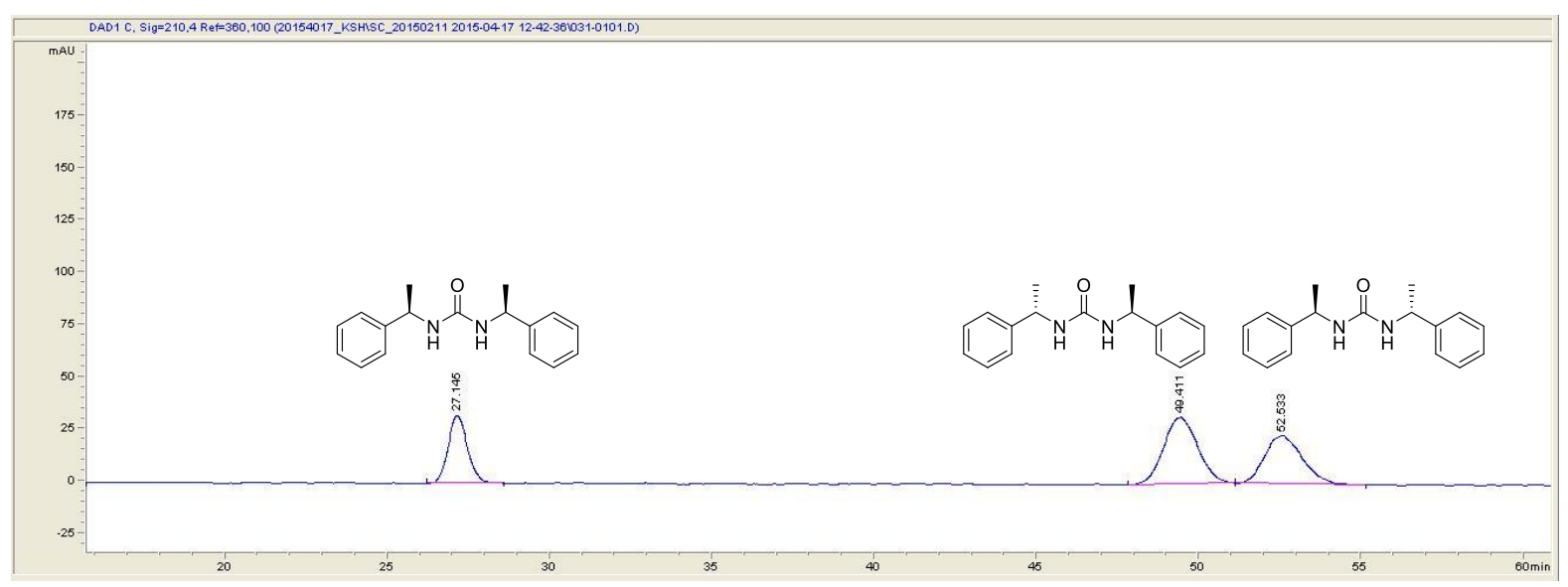

Figure S2. The LC chart for 1,3-bis((S)-1-phenylethyl)urea

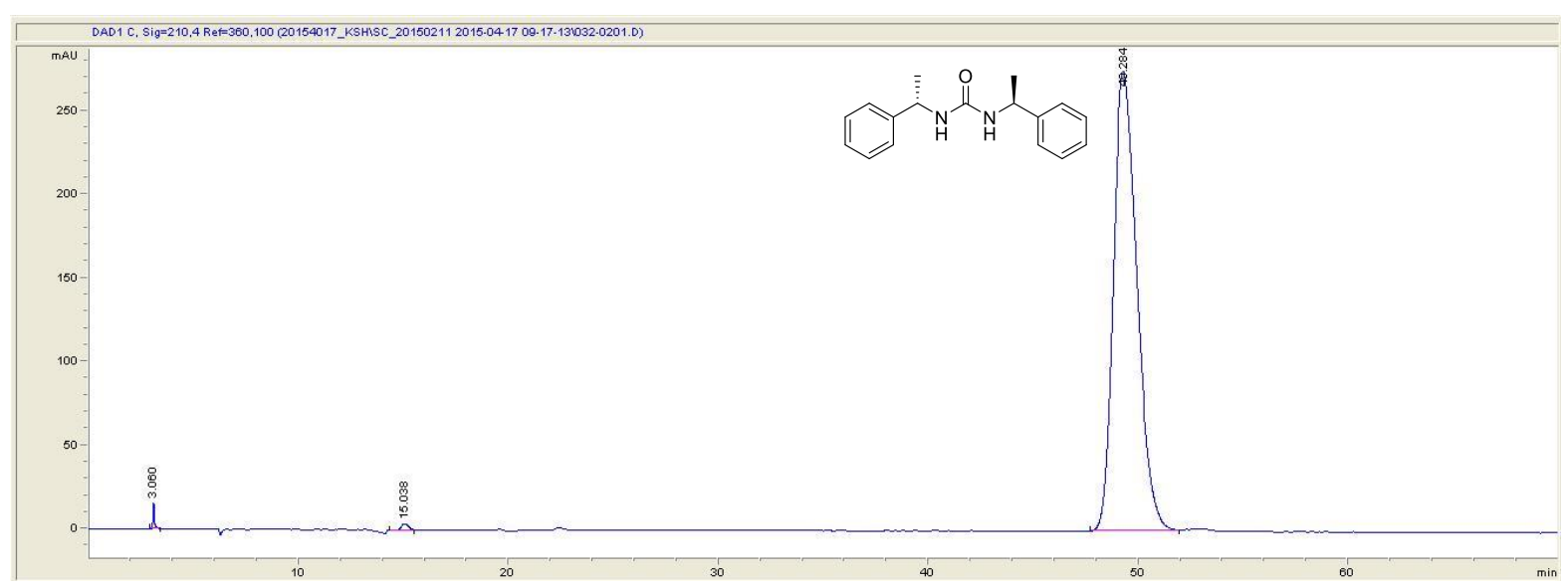




\section{References}

(1) Han, Z. B.; Rong, L. C.; Wu, J.; Zhang, L.; Wang, Z.; Ding, K. L. Angew. Chem. Int. Ed. 2012, 51, 13041-13045.

(2) Kang, B.; Hong, S. H. Adv. Synth. Catal. 2015, 357, 834-840.

(3) Mizuno, T.; Mihara, M.; Iwai, T.; Ito, T.; Ishino, Y. Synthesis 2006, 2825-2830.

(4) Artuso, E.; Degani, I.; Fochi, R.; Magistris, C. Synthesis 2007, 3497-3506.

(5) Mizuno, T.; Mihara, M.; Nakai, T.; Iwai, T.; Ito, T. Synthesis 2007, 3135-3140.

(6) McCusker, J. E.; Main, A. D.; Johnson, K. S.; Grasso, C. A.; McElwee-White, L. J. Org. Chem. 2000, 65, 5216-5222.

(7) Park, J. H.; Yoon, J. C.; Chung, Y. K. Adv. Synth. Catal. 2009, 351, 1233-1237.

(8) Zhang, M.; Imm, S.; Bähn, S.; Neubert, L.; Neumann, H.; Beller, M. Angew. Chem. Int. Ed. 2012, 51, 3905-3909.

(9) Orito, K.; Miyazawa, M.; Nakamura, T.; Horibata, A.; Ushito, H.; Nagasaki, H.; Yuguchi, M.; Yamashita, S.; Yamazaki, T.; Tokuda, M. J. Org. Chem. 2006, 71, 5951-5958.

(10) Peterson, S. L.; Stucka, S. M.; Dinsmore, C. J. Org. Lett. 2010, 12, 1340-1343. 
NMR Spectra

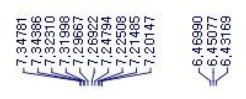

縞

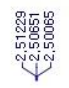
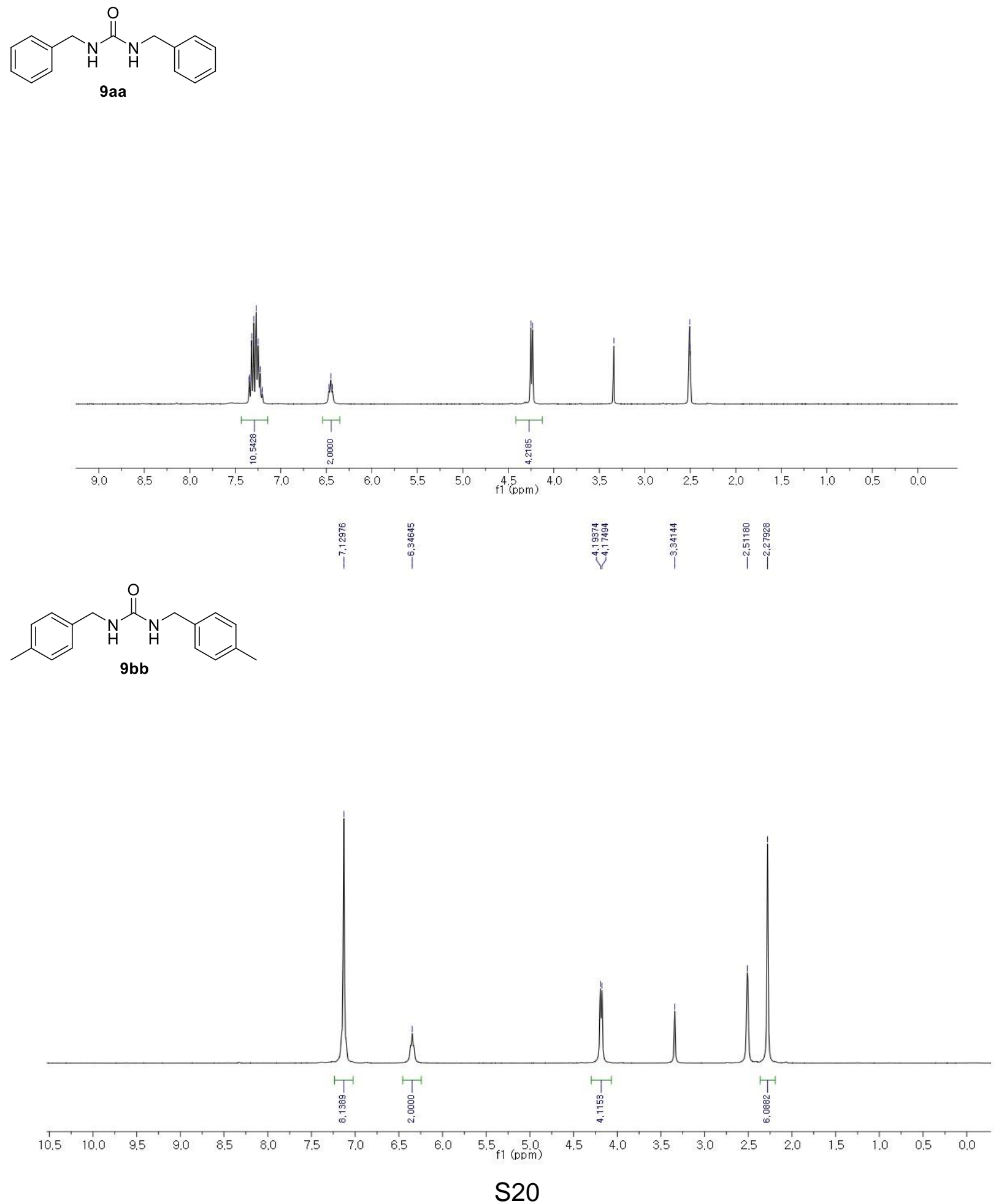


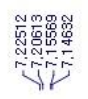

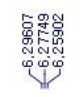

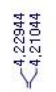

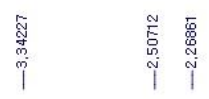
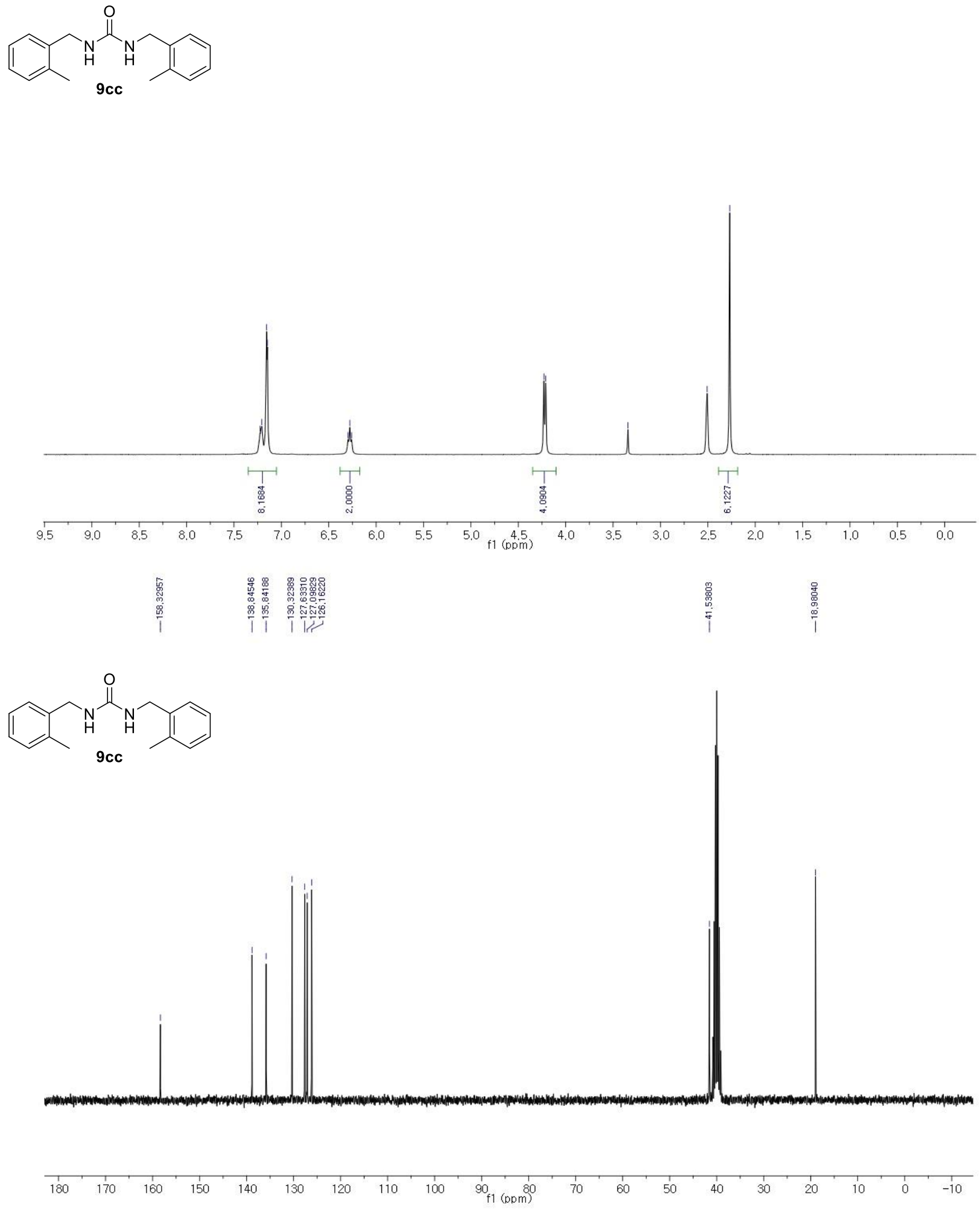

S21 
<smiles>COc1ccc(CNC(=O)NCc2ccc(OC)cc2)cc1</smiles>

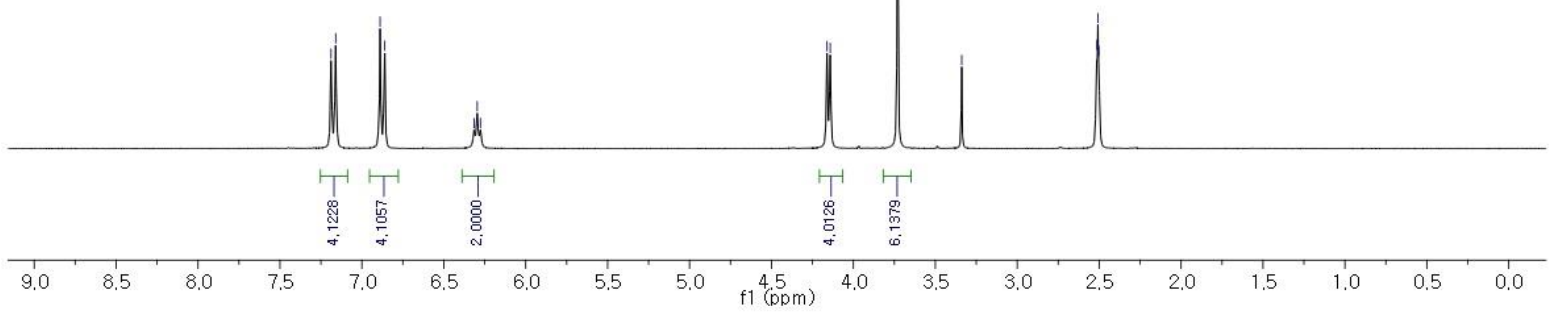

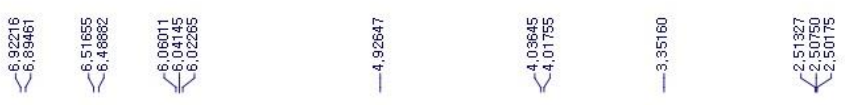<smiles>Nc1ccc(CNC(=O)NCc2ccc(N)cc2)cc1</smiles>

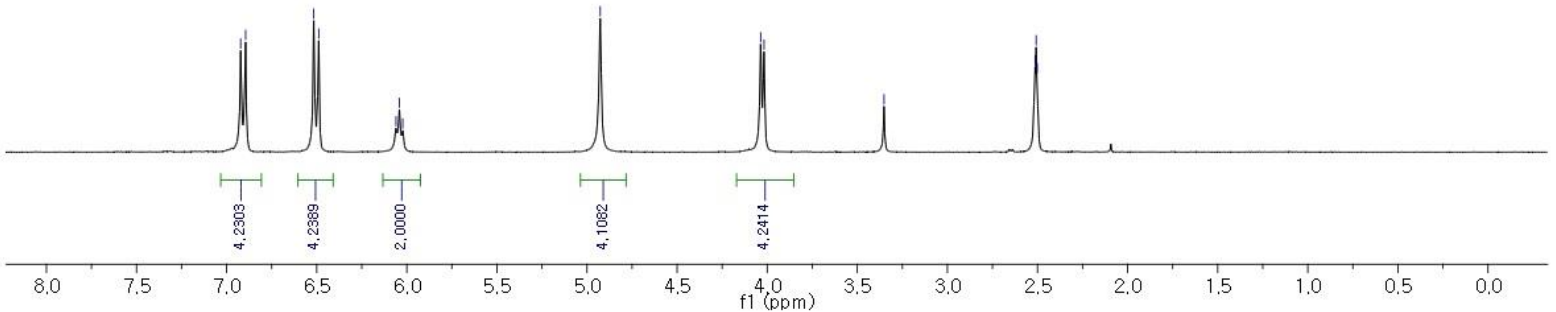



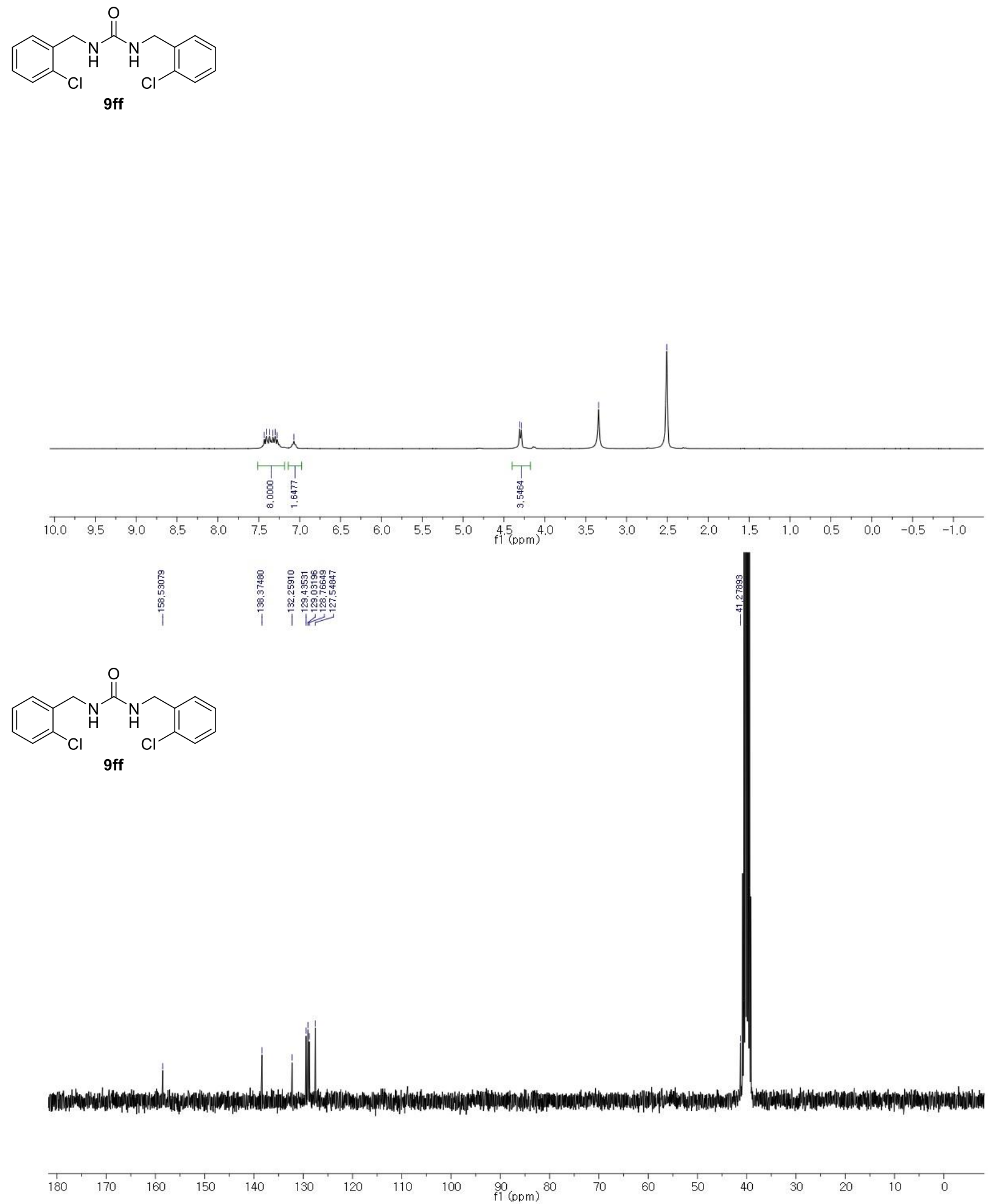

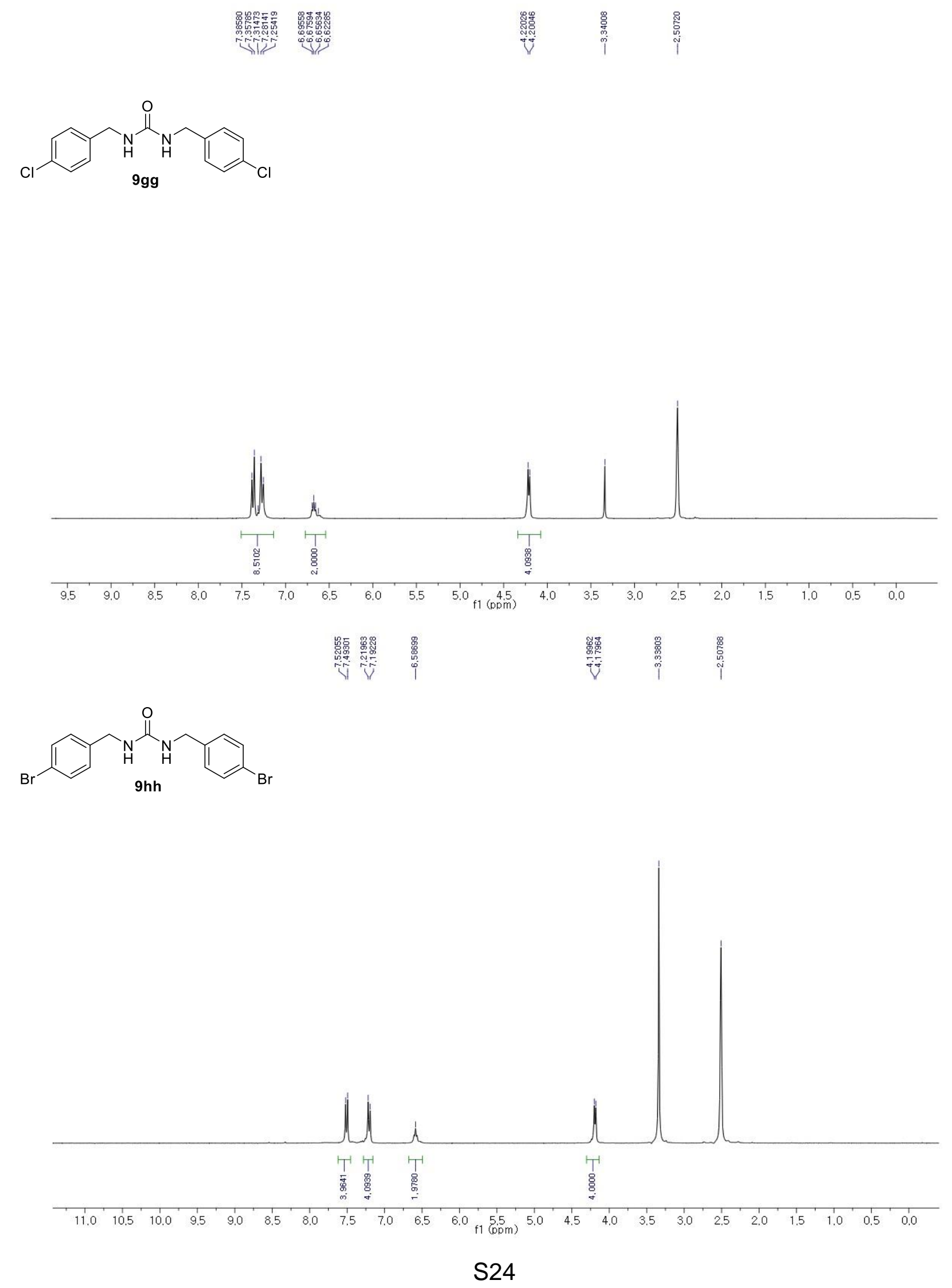


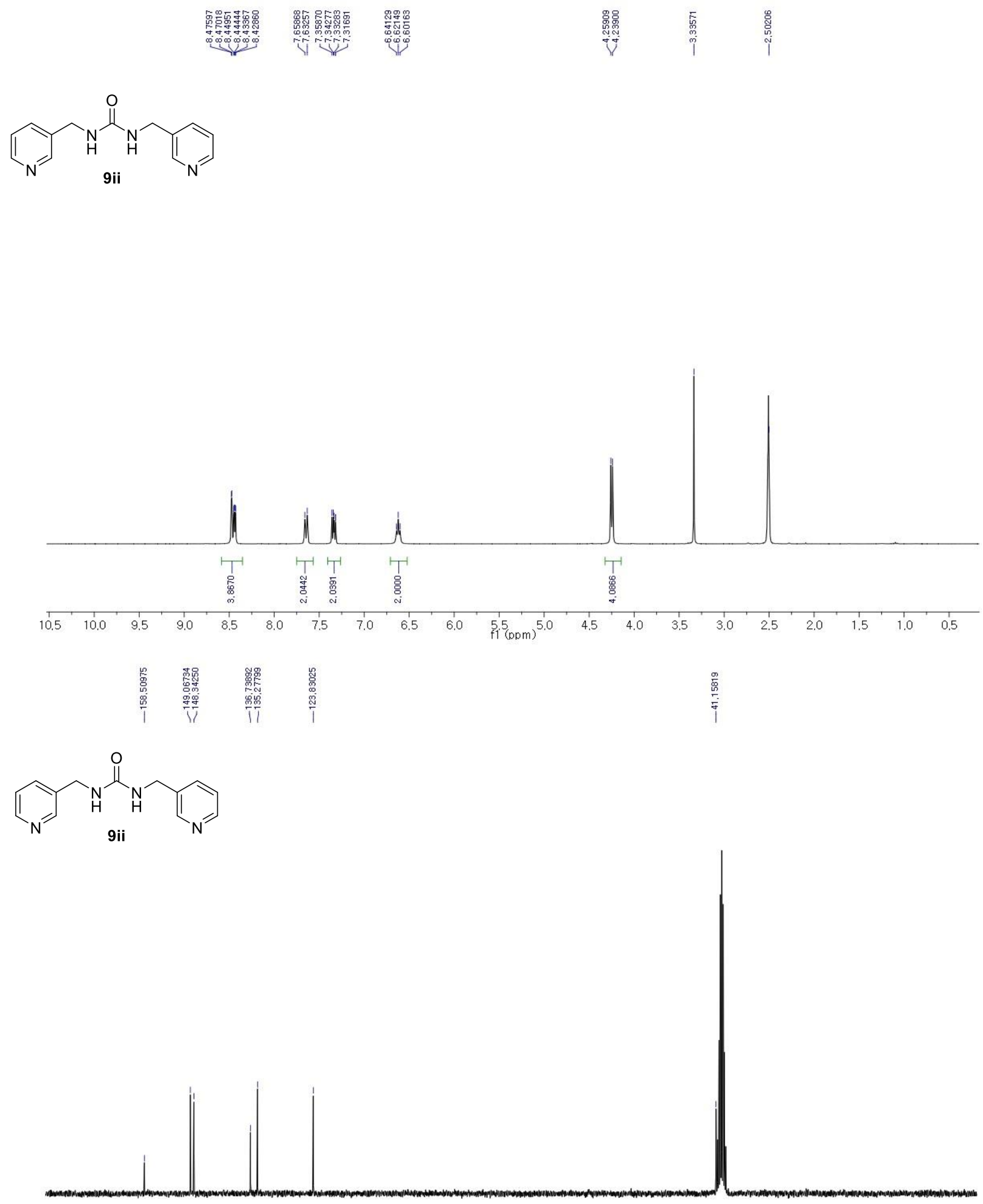

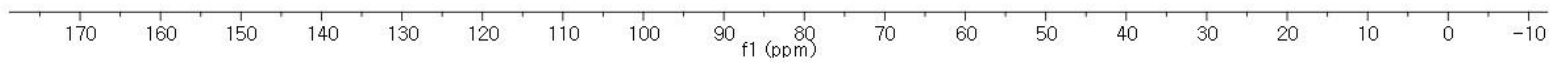



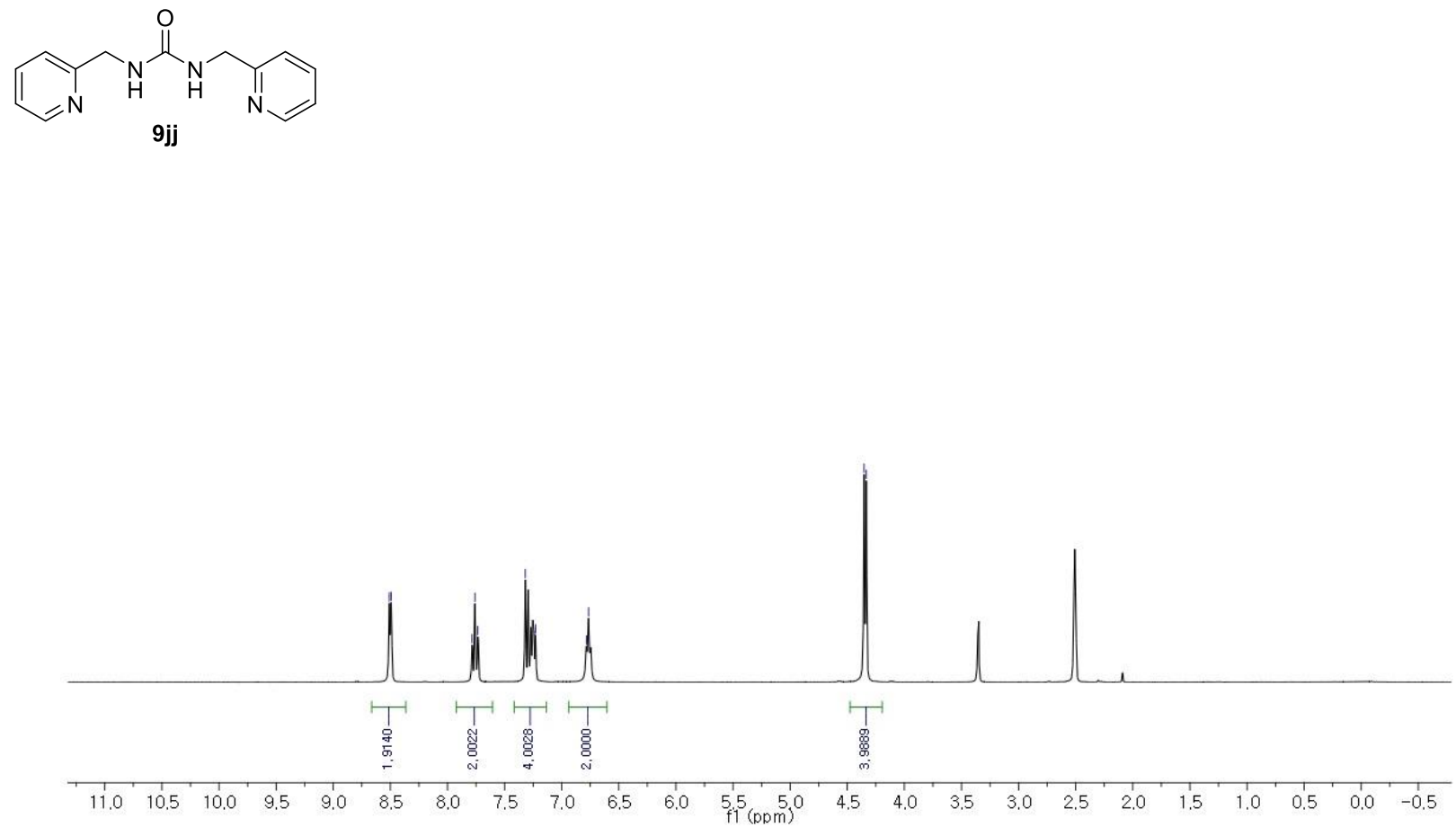

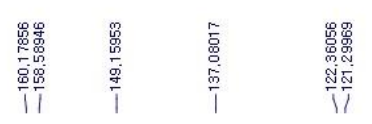
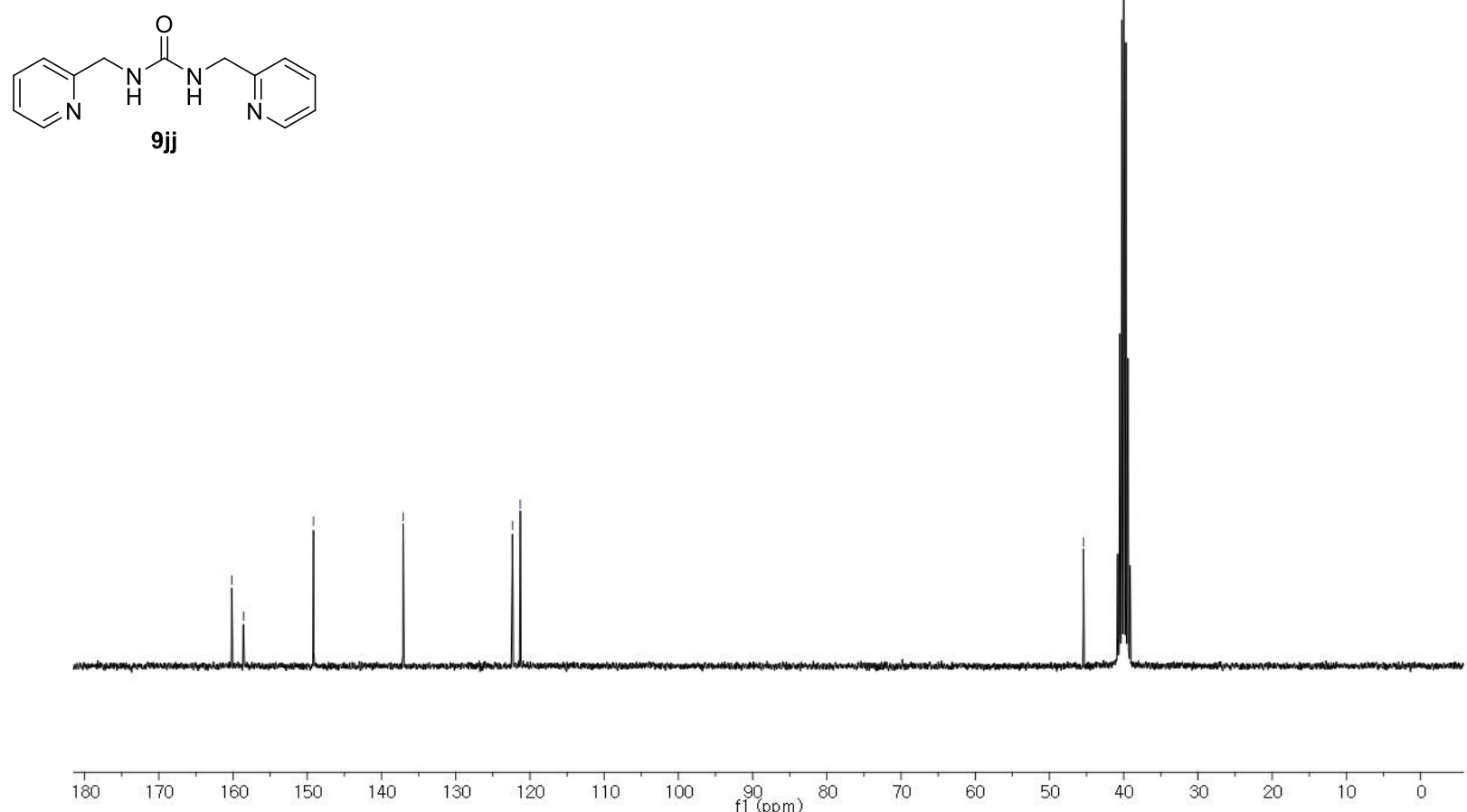

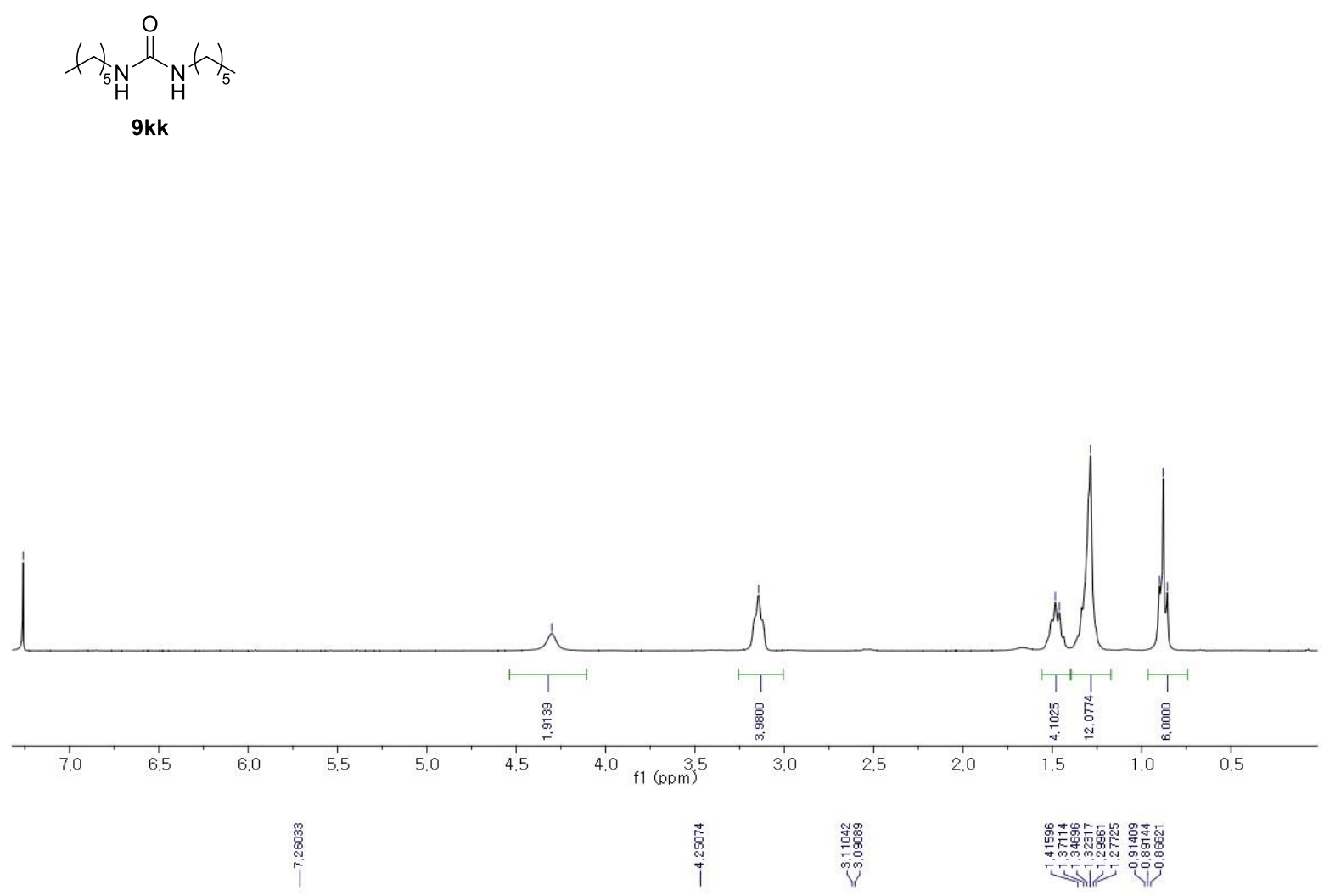

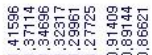
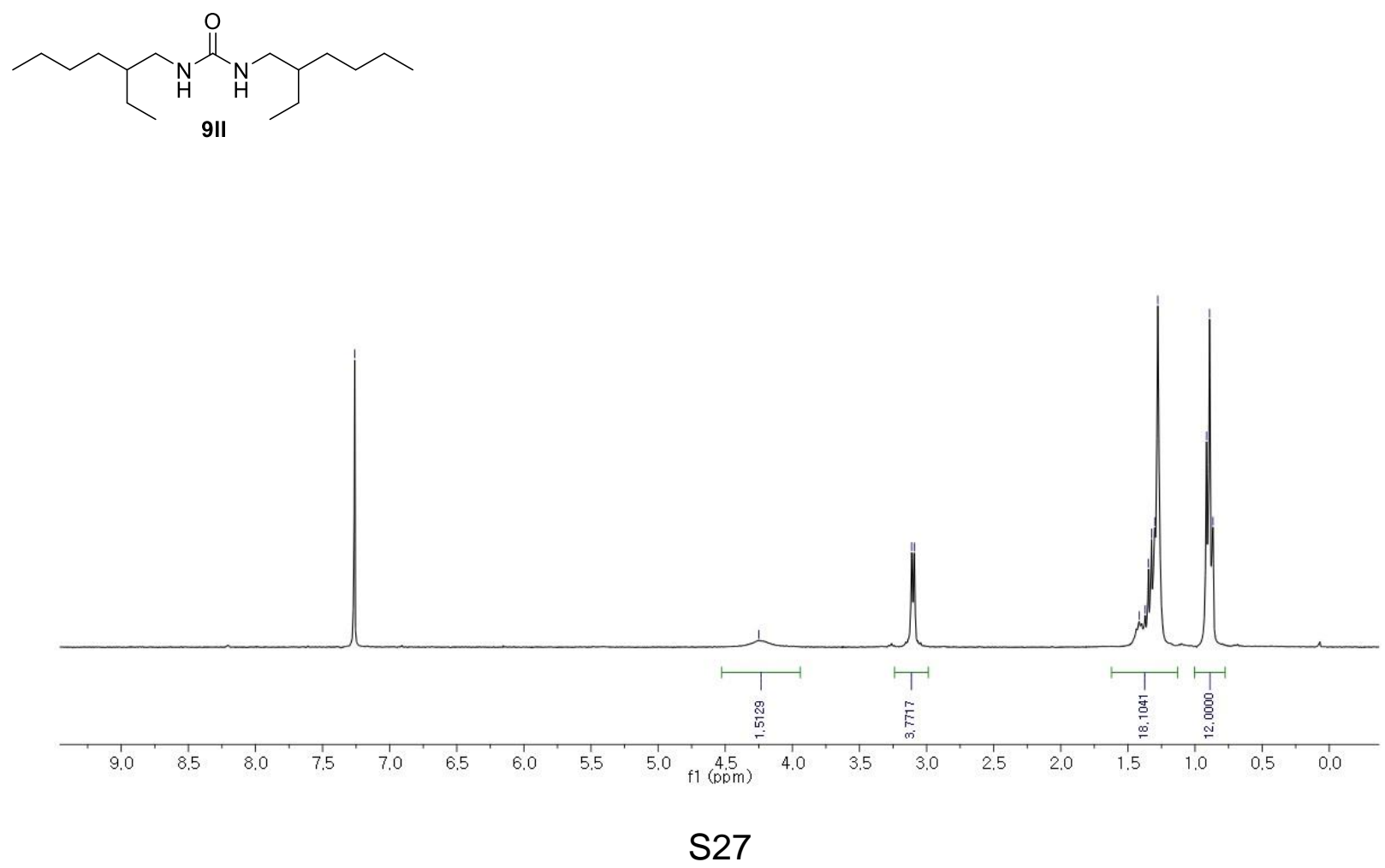

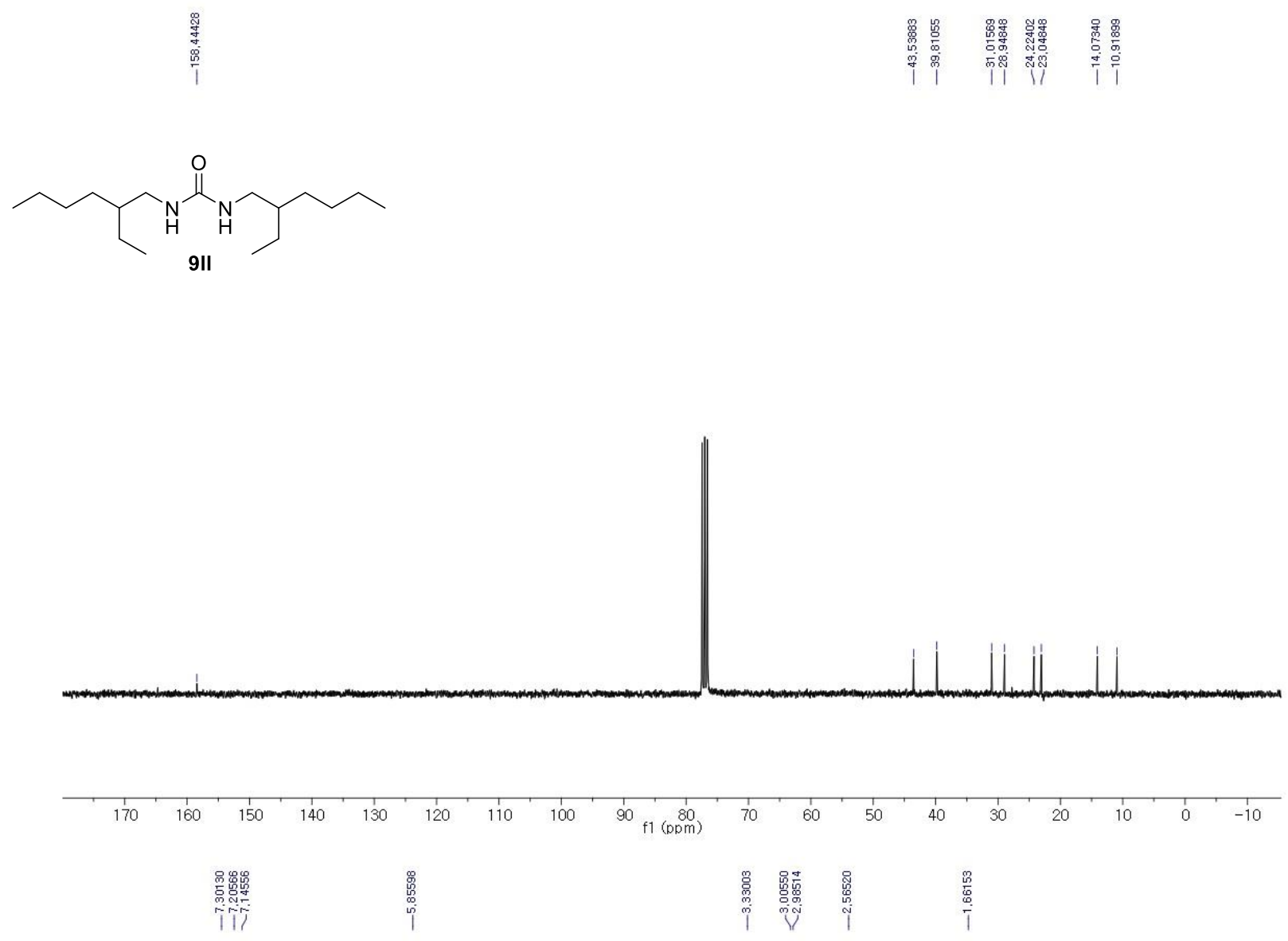<smiles>O=C(NCCCc1ccccc1)NCCCc1ccccc1</smiles>

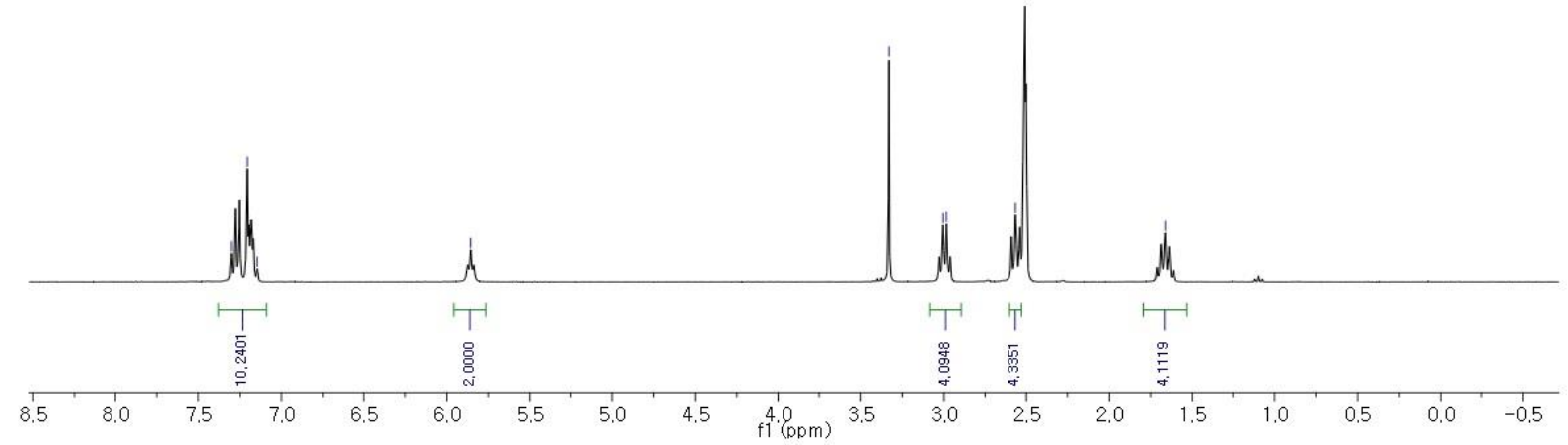



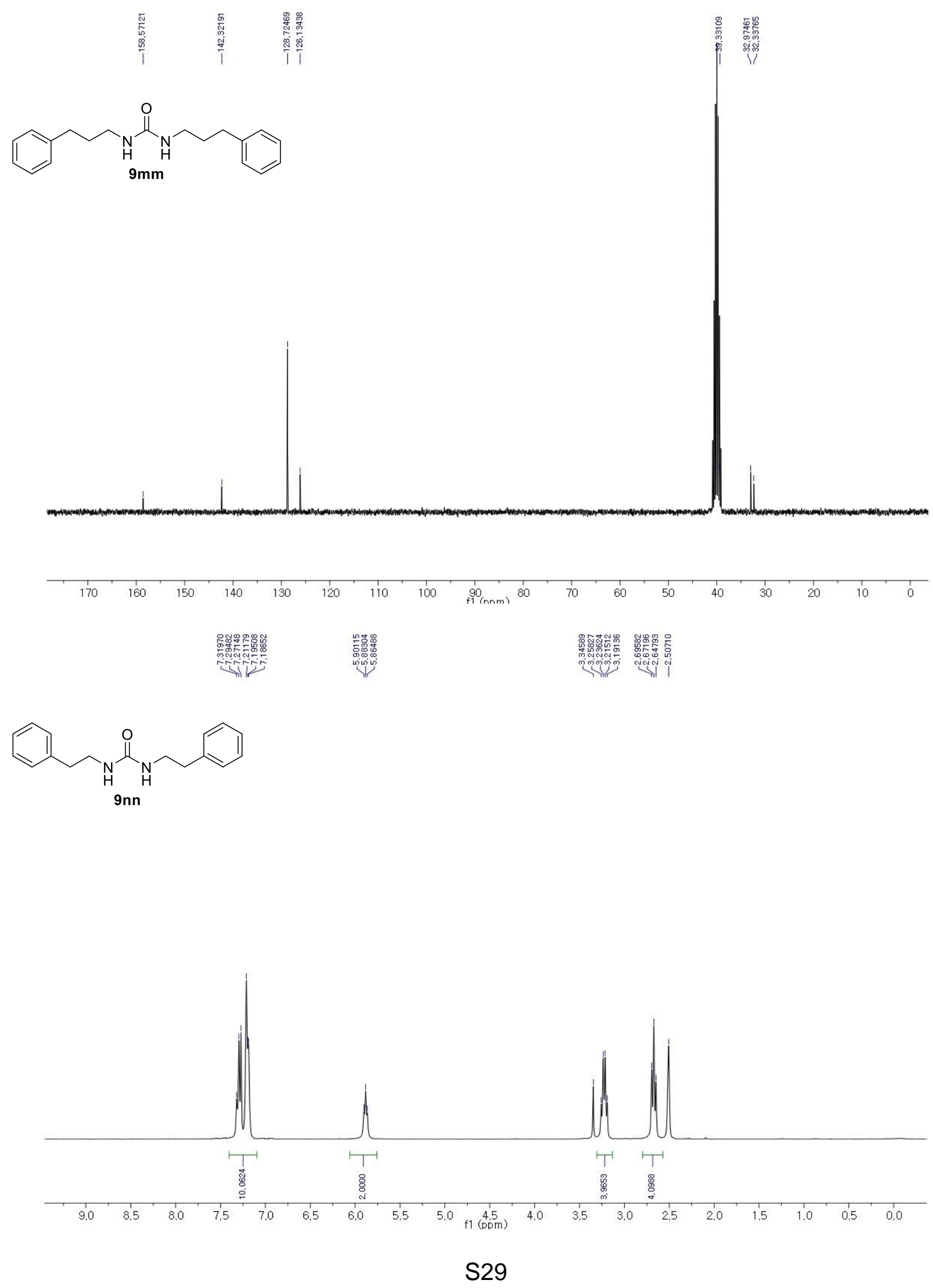


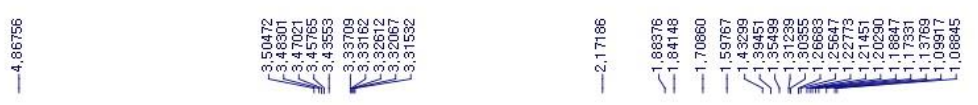

$\overbrace{900}$
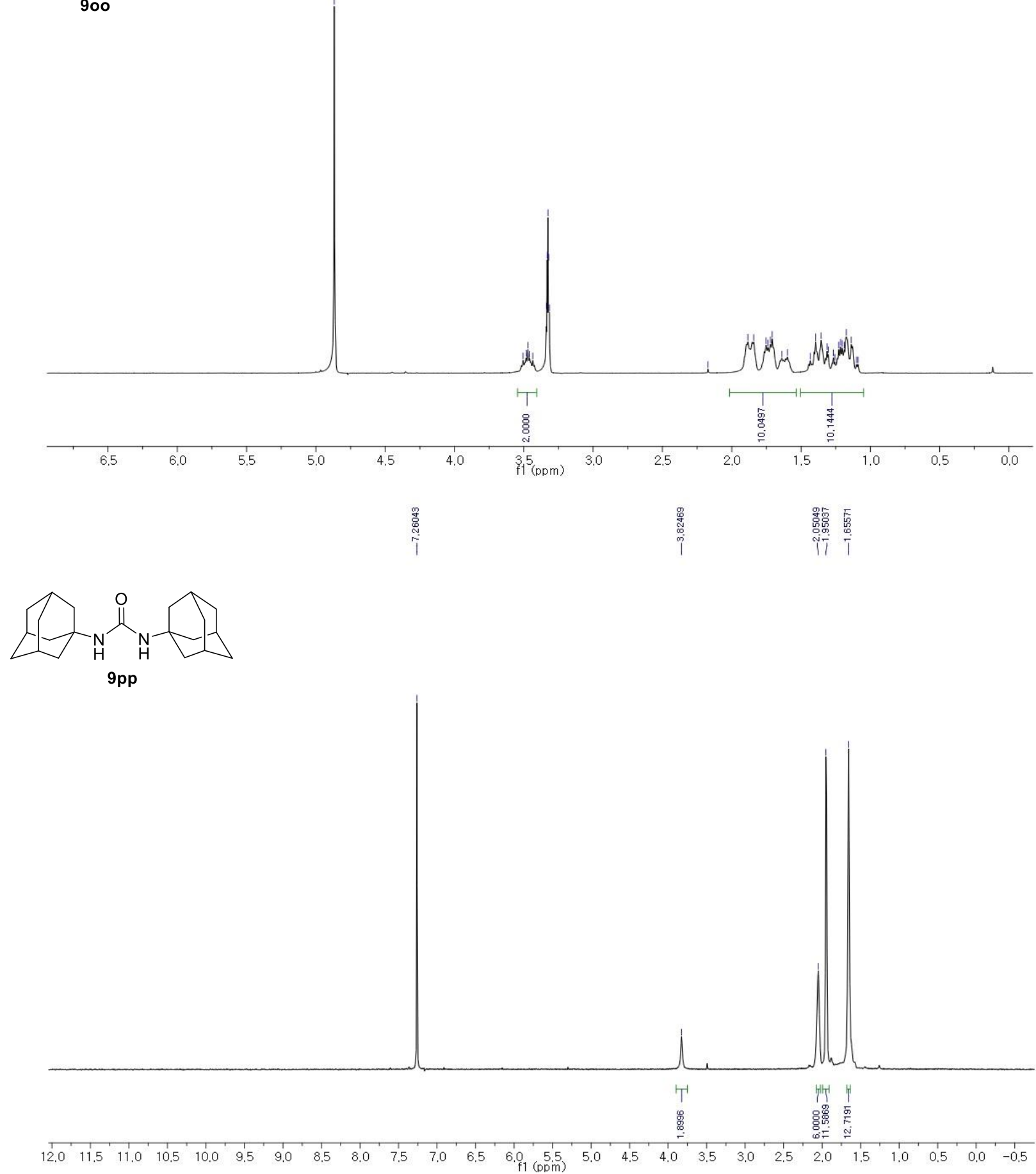

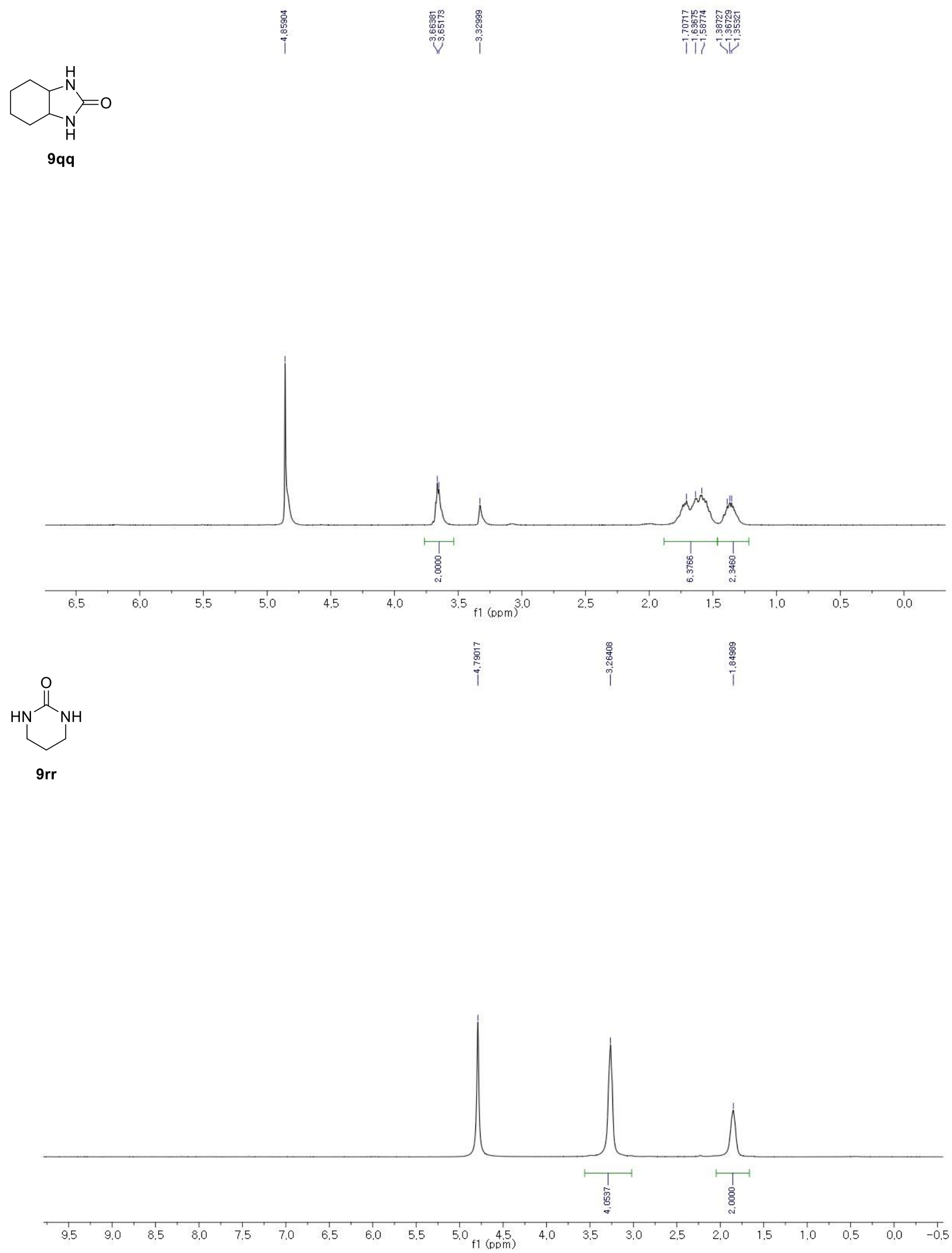

S31 

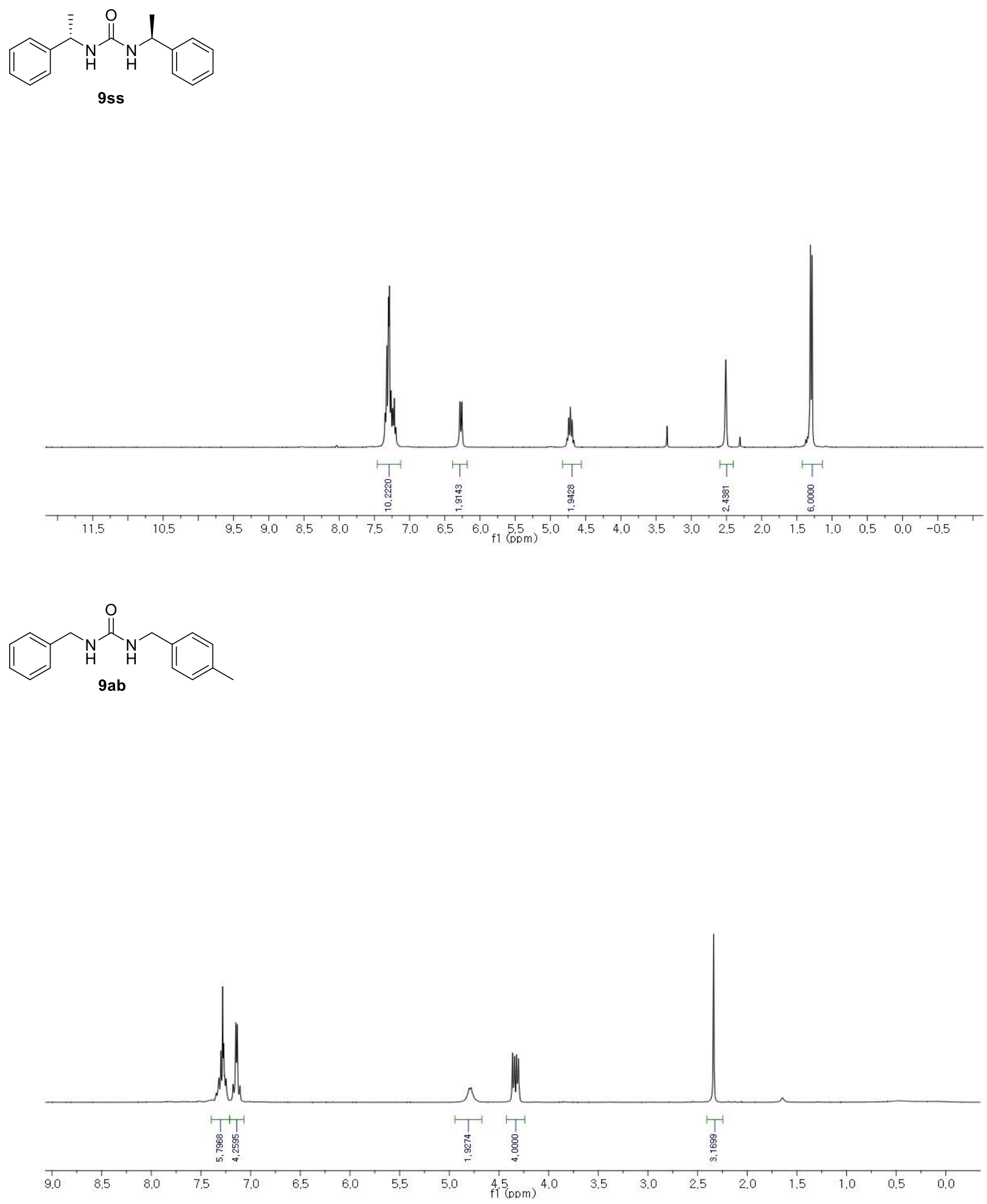

S32 

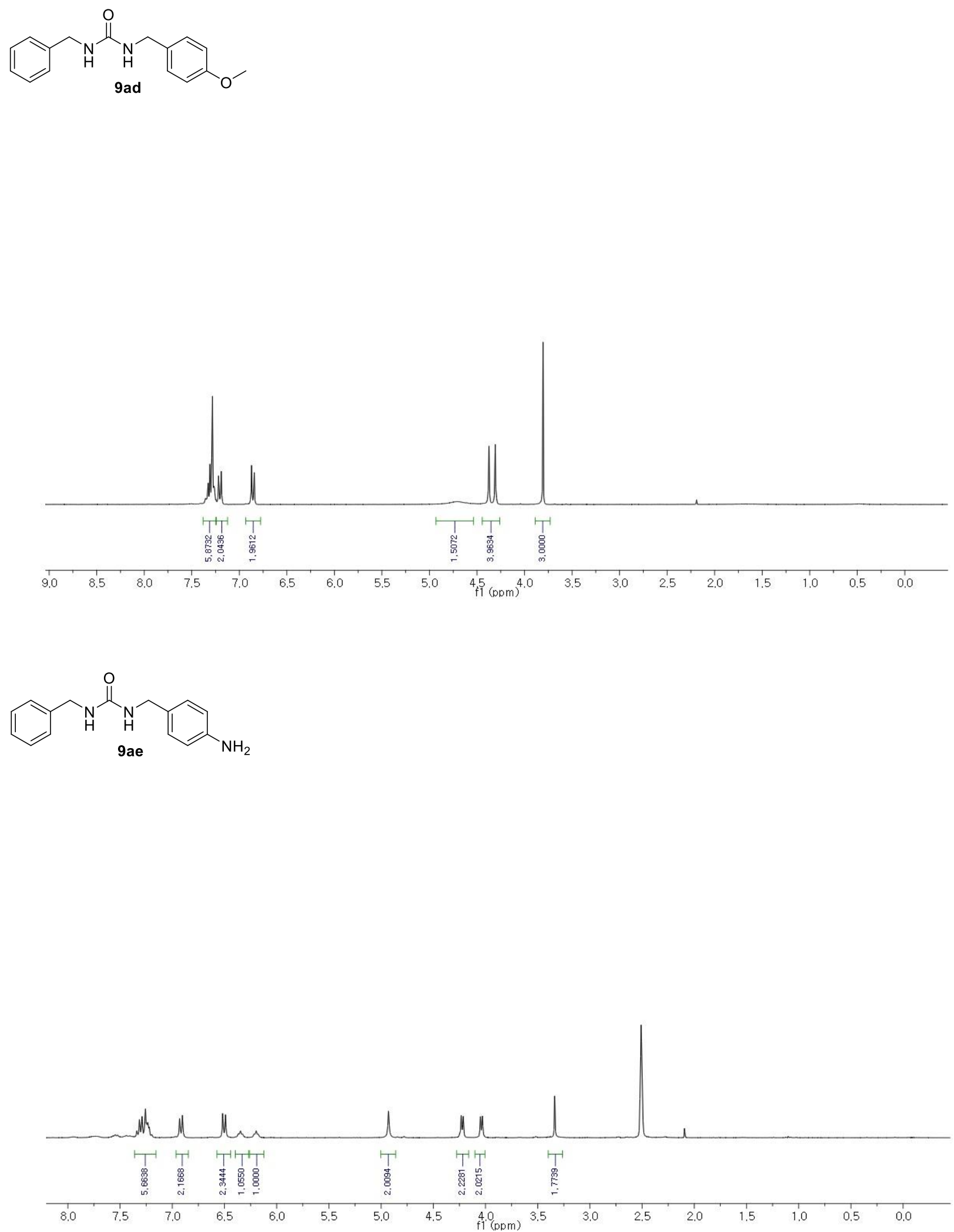

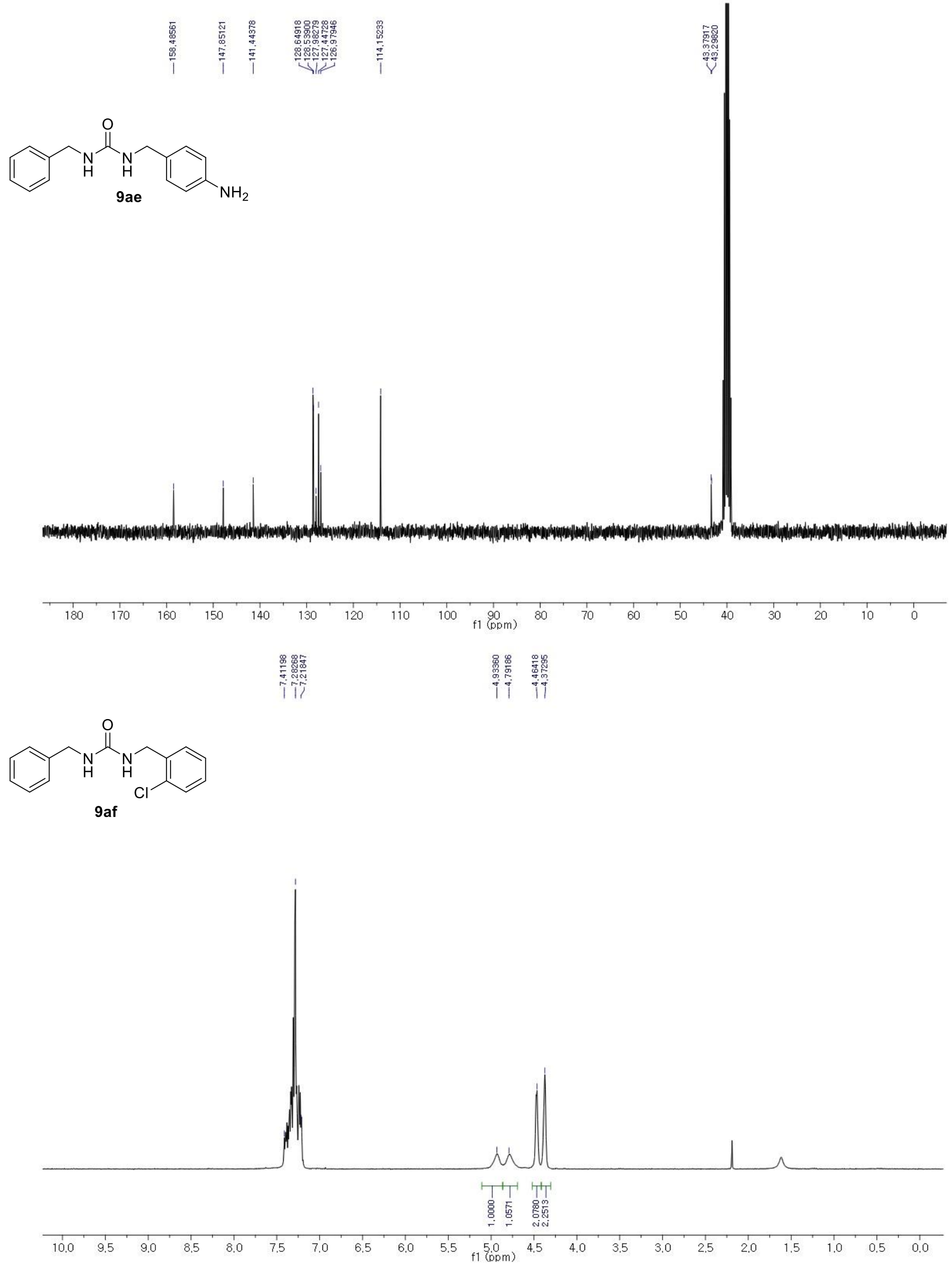


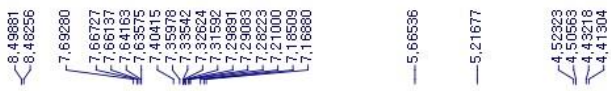<smiles>O=C(NCc1ccccc1)NCc1ccccn1</smiles>

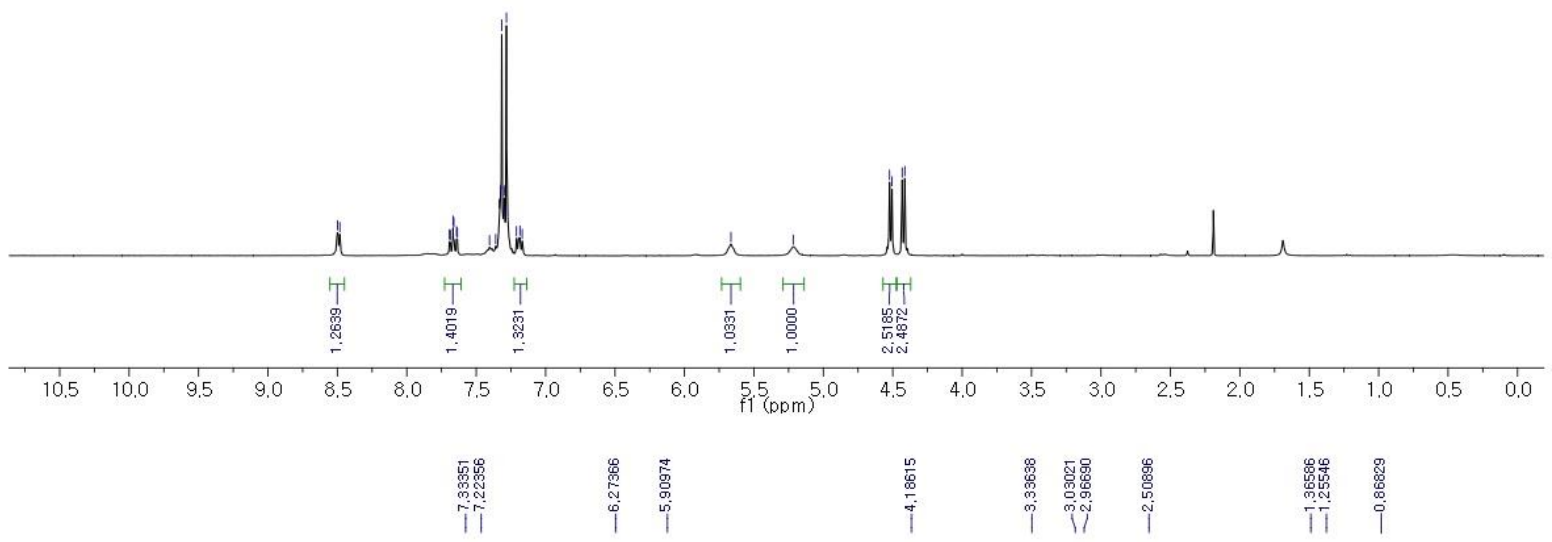<smiles>CCCCCCNC(=O)NCc1ccccc1</smiles>

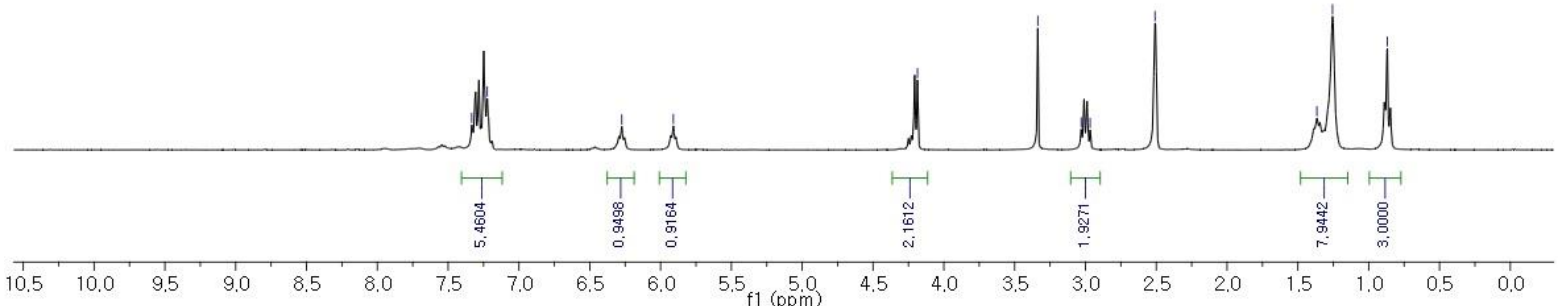



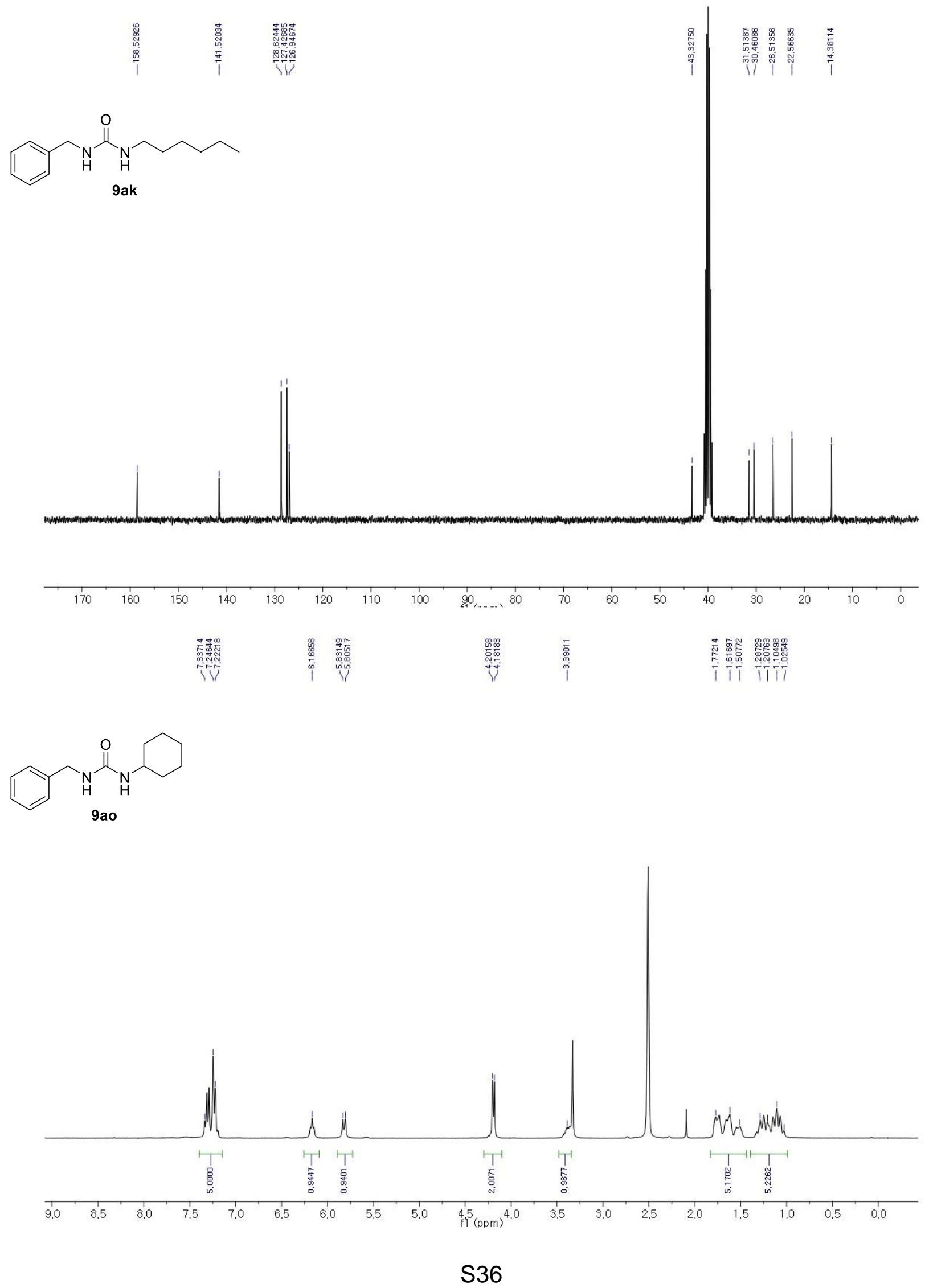

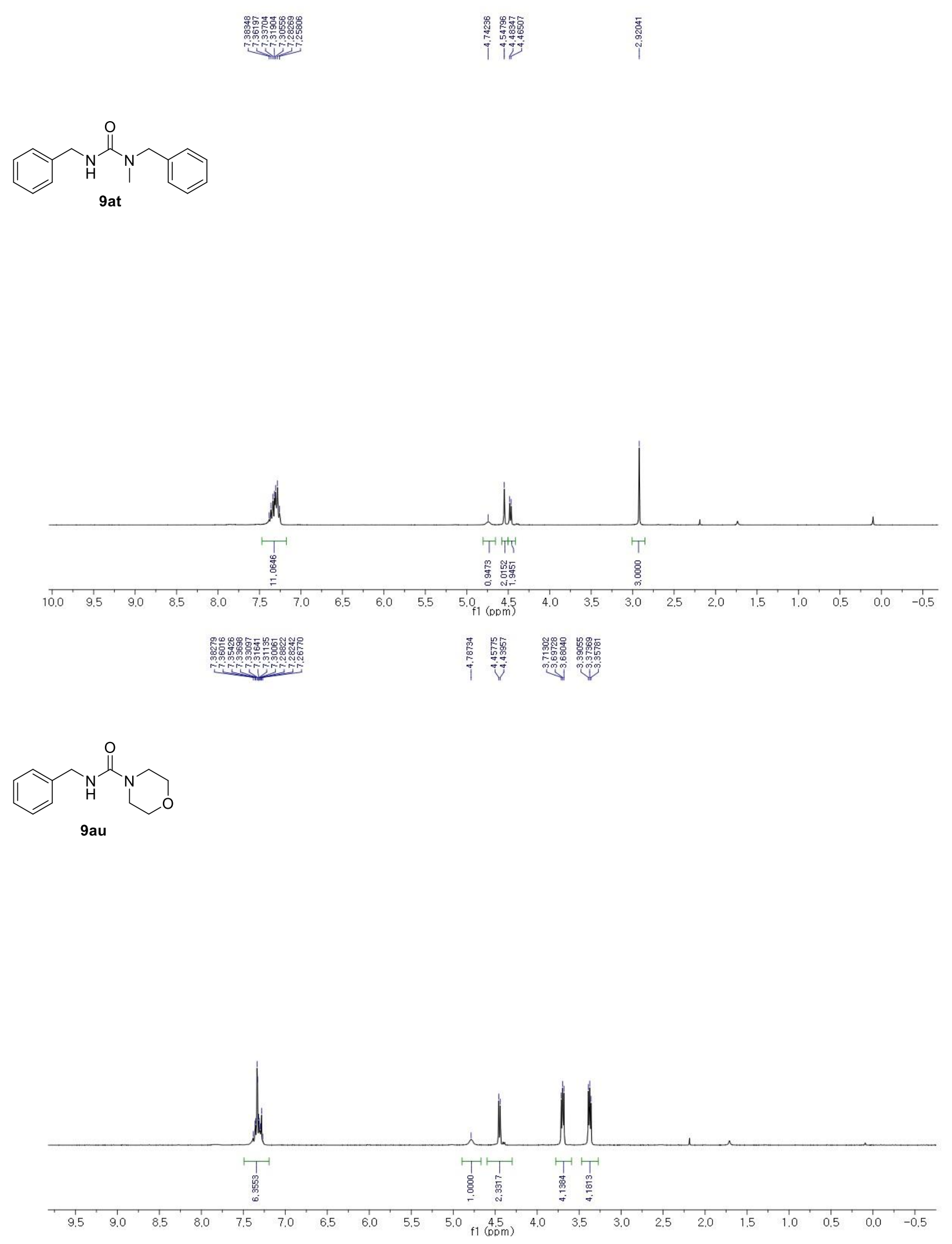


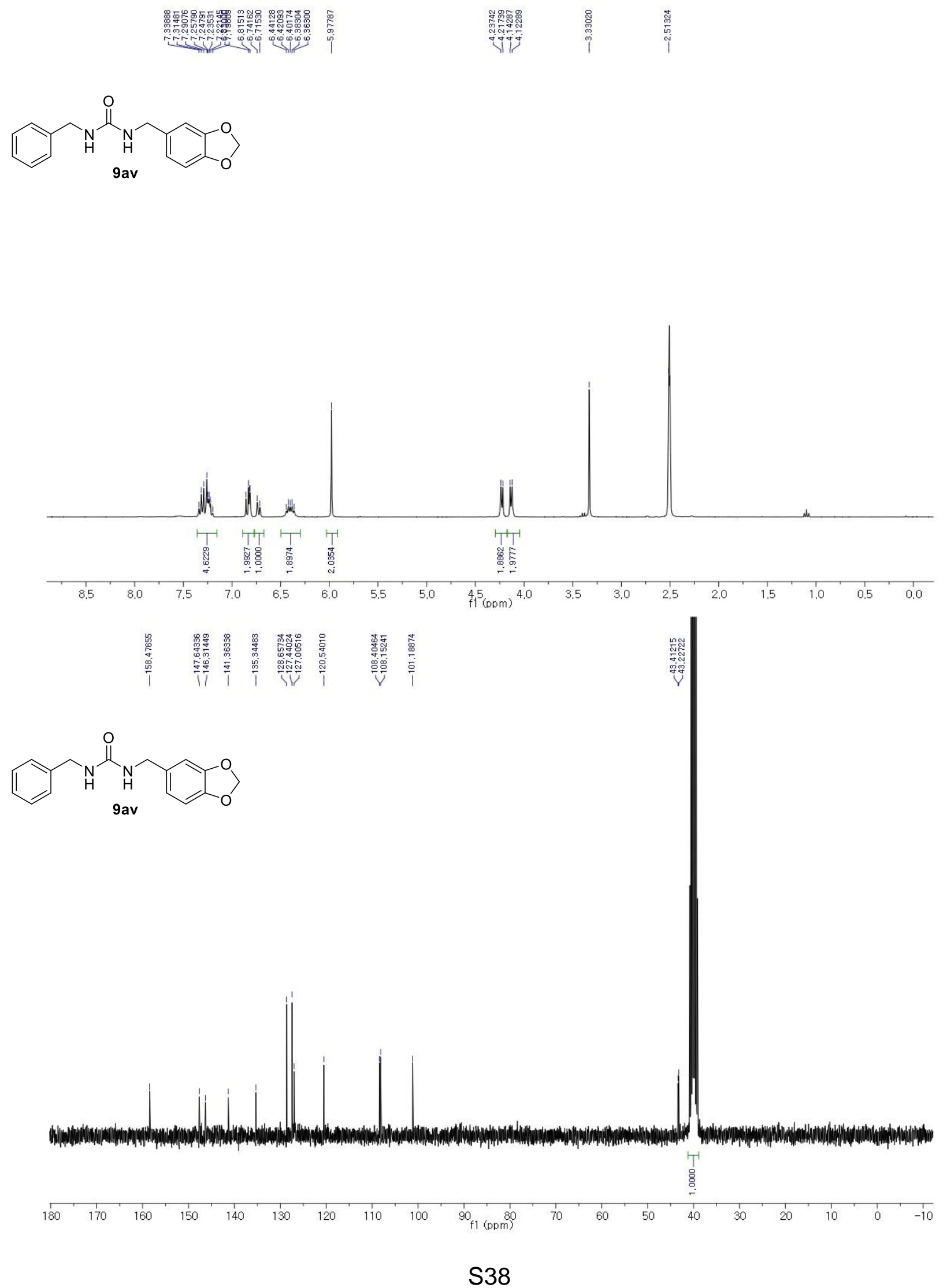




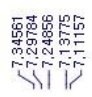

高

熵

0
0
0
0
0
0
0

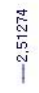
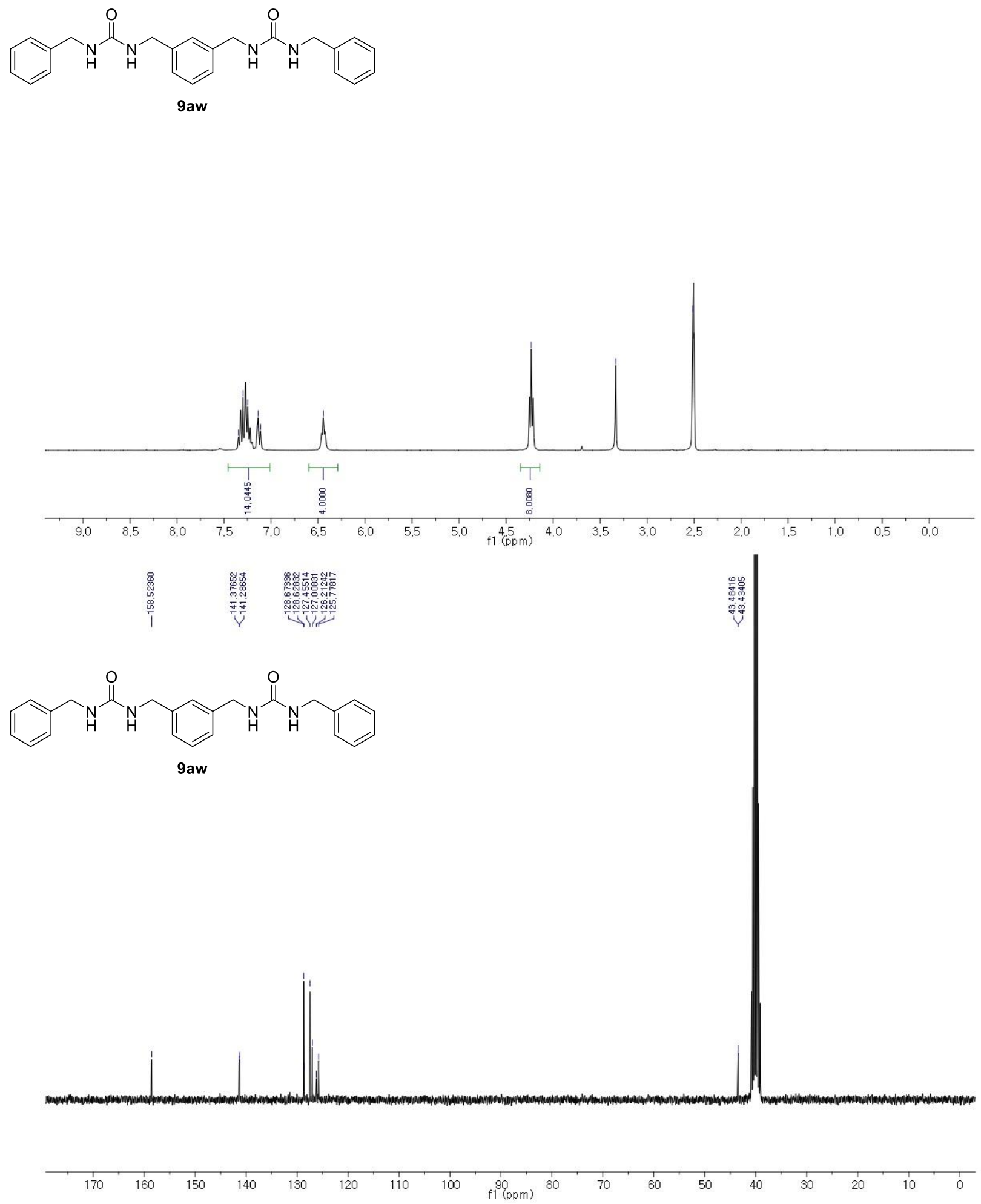

S39 


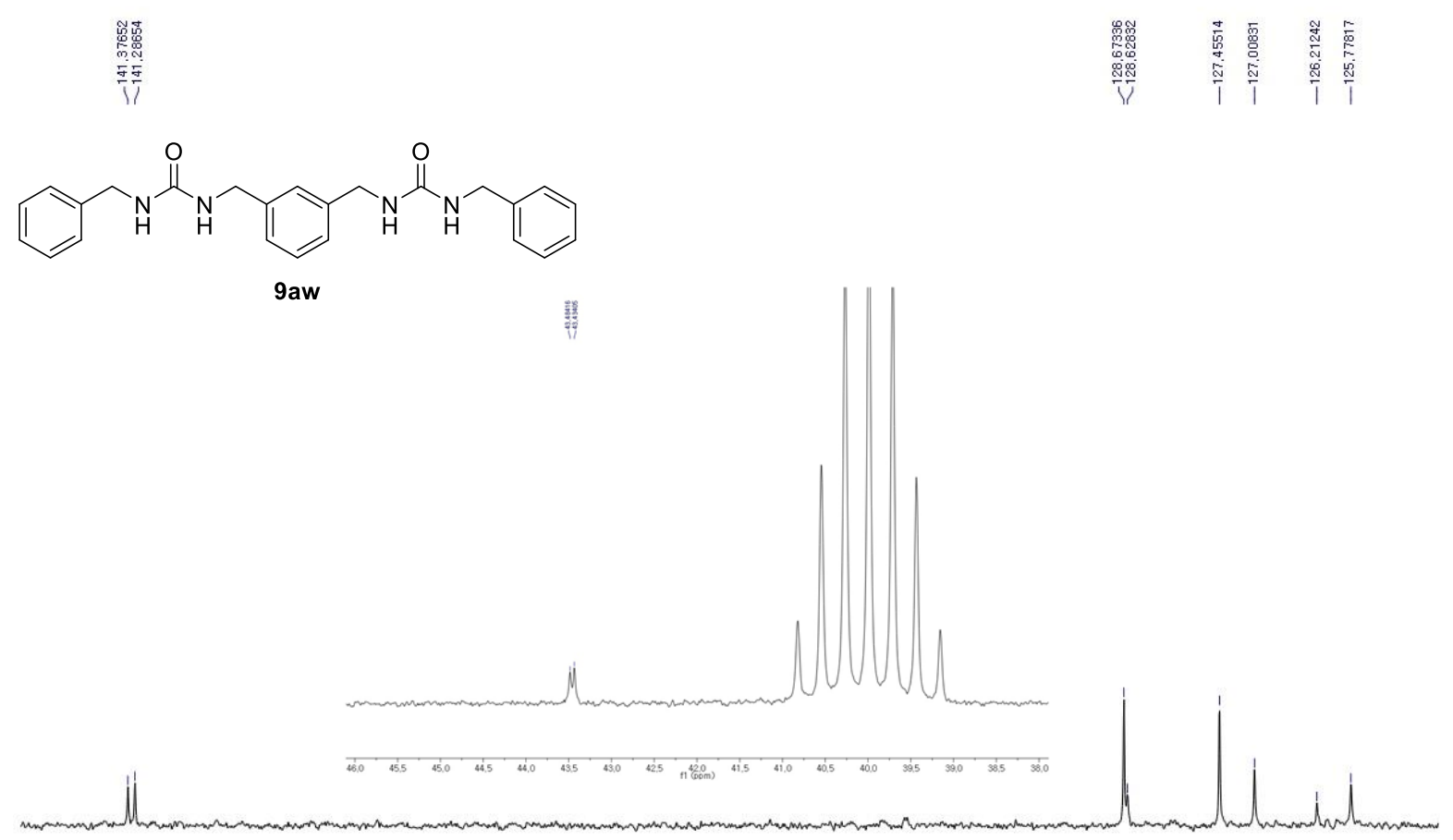

\begin{tabular}{|c|c|c|c|c|c|c|c|c|c|c|c|c|c|c|c|}
\hline 142 & 141 & 140 & 139 & 138 & 137 & 136 & 135 & ${ }^{134}{ }^{1}(\mathrm{ppm})^{133}$ & 132 & 131 & 130 & 129 & 128 & 127 & 126 \\
\hline
\end{tabular}

